\title{
Wave scattering by a floating porous elastic plate of arbitrary shape: a semi-analytical study
}

\section{Zheng, Siming}

http://hdl.handle.net/10026.1/15229

\author{
10.1016/j.jfluidstructs.2019.102827 \\ Journal of Fluids and Structures \\ Elsevier
}

All content in PEARL is protected by copyright law. Author manuscripts are made available in accordance with publisher policies. Please cite only the published version using the details provided on the item record or document. In the absence of an open licence (e.g. Creative Commons), permissions for further reuse of content should be sought from the publisher or author. 
Title:

Wave scattering by a floating porous elastic plate of arbitrary shape: A semi-analytical study

Author names and affiliations:

Siming Zheng ${ }^{\mathrm{a}}$, Mike H. Meylan ${ }^{\mathrm{b}}$, Lin Fan ${ }^{\mathrm{c}}$, Deborah Greaves ${ }^{\mathrm{a}}$, Gregorio Iglesias ${ }^{\mathrm{d}, \mathrm{a}}$

a School of Engineering, University of Plymouth, Drake Circus, Plymouth PL4 8AA, UK

b School of Mathematical and Physical Sciences, The University of Newcastle, Callaghan 2308, Australia

c Development Research Center of the Ministry of Water Resources, Beijing 100036, China

d MaREI, Environmental Research Institute \& School of Engineering, University College Cork, Ireland

E-mail address:

Siming Zheng ${ }^{\mathrm{a}} \quad$ siming.zheng@plymouth.ac.uk

Mike H. Meylan ${ }^{\mathrm{b}}$ mike.meylan@newcastle.edu.au

Lin Fan ${ }^{\mathrm{c}}$ : 1-fan11@tsinghua.org.cn

Deborah Greaves $^{\mathrm{a}} \quad$ deborah.greaves@plymouth.ac.uk

Gregorio Iglesias $^{\mathrm{d}, \mathrm{a}}$ gregorio.iglesias@ucc.ie

Corresponding author: Siming Zheng

E-mail address: siming.zheng@plymouth.ac.uk

Received 11 July 2019, Revised 23 October 2019, Accepted 29 November 2019, Available online 9 December 2019.

https://doi.org/10.1016/j.jfluidstructs.2019.102827 


\title{
Wave scattering by a floating porous elastic plate of arbitrary shape: a semi-analytical study
}

\author{
Siming Zhenga,*, Michael H. Meylan ${ }^{\mathrm{b}}$, Lin Fan ${ }^{\mathrm{c}}$, Deborah Greaves ${ }^{\mathrm{a}}$, Gregorio \\ Iglesias $^{\mathrm{d}, \mathrm{a}}$ \\ ${ }^{a}$ School of Engineering, University of Plymouth, Drake Circus, Plymouth PL4 8AA, UK \\ ${ }^{b}$ School of Mathematical and Physical Sciences, The University of Newcastle, Callaghan \\ 2308, Australia \\ ${ }^{c}$ Development Research Center of the Ministry of Water Resources, Beijing 100036, China \\ ${ }^{d}$ MaREI, Environmental Research Institute 83 School of Engineering, University College \\ Cork, Ireland
}

\begin{abstract}
In this paper, a semi-analytical model based on linear potential flow theory and an eigenfunction expansion method is developed to study wave scattering by a porous elastic plate with arbitrary shape floating in water of finite depth. The water domain is divided into the interior and exterior regions, corresponding to the domain beneath the plate and the rest extending towards infinite distance horizontally, respectively. The unknown coefficients in the potential expressions are determined by satisfying the continuity conditions for pressure and velocity at the interface of the two regions, together with the conditions for the motion/force at the edge of the plate, where the Fourier series expansion method is employed to deal with the terms associated with the radius function. A plate with three shapes - circular, cosine and elliptical - and three edge conditions are considered. We find that the largest deflection of the plate with a simply supported edge and a clamped edge is more sensitive to the change in porosity when the porosity is small. The power dissipated by an elliptical plate with its major axis perpendicular to the incident wave direction is the largest among the case studies for the majority of the porosity values tested.
\end{abstract}

Keywords: Wave-structure interaction, Potential flow theory, Porous elastic

*Corresponding author. Email: siming.zheng@plymouth.ac.uk 
plate, Eigenfunction expansion method, Arbitrary shape

\section{Introduction}

It is widely known that porous structures work well in dissipating wave power and attenuating wave motion (Chwang and Chan, 1998). Porous structures can be typically horizontal, vertical or inclined plates. A horizontal porous plate allows the free exchange of water or marine animals for the benefit of the environment; therefore, water-wave interaction with horizontal porous plates, in cases such as mobile offshore bases, floating breakwaters and floating vegetated platforms (see Chwang and Chan (1998); Wang et al. (2010); Koley et al. (2018); Chen et al. (2016) for details), has been investigated by many researchers.

$\mathrm{Yu}$ and Chwang (1994) used the boundary element method (BEM) to study wave diffraction by a horizontally submerged porous plate. They demonstrated that a plate with the proper porosity could significantly attenuate the reflection while keeping the transmission at a low level. Yip and Chwang (1998) proposed active control of a submerged porous plate by adjusting the magnitude and phase of pitching and analyzed its characteristics by means of an analytical model. It was shown that the wave transformation due to pitching reduced rapidly with increase in porosity. Evans and Peter (2011) presented an explicit expression for the reflection coefficient when a plane wave was obliquely incident upon a semi-infinite submerged porous plate by means of the Wiener-Hopf technique. An alternative analytical solution for wave interaction with a submerged horizontal porous-plate breakwater was proposed by Liu and Li (2011) by using non-standard eigenfunctions in the plate region. Therefore, they avoided finding the complex roots of a special dispersion relation in the porous-plate region. The problem of wave scattering by a thin submerged porous plate was reduced to a hypersingular integral equation and then solved with the employment of polynomial expansions by Gayen and Mondal (2014). This method is very powerful for solving submerged plate problems in two dimensions. Apart from porous plates, flexible plates were regarded as an alternative, effective, and inexpen- 
sive wave barriers. Cho and Kim (1998) investigated the interaction of waves with a horizontal flexible membrane in the context of $2 \mathrm{D}$ linear hydro-elastic theory. They developed an analytical model for solving the wave diffraction and radiation problem using an eigenfunction expansion method. It was shown that a properly designed horizontal flexible membrane could be a very effective wave barrier. Their model was later extended to study wave interaction with a horizontal porous flexible membrane (Cho and Kim, 2000). The analytical results were in good agreement with the BEM-based numerical results. It was revealed that the wave-blocking performance of the membrane could be further enhanced by adding proper porosity. The performance of a submerged flexible porous plate in both cases of deep and shallow water was analyzed by Mohapa40 tra et al. (2018). A flexible plate with both of its faces bonded by piezoelectric layers may work as a piezoelectric plate wave energy converter (WEC) Renzi, 2016). The tension variations at the plate-water interface induced by wave motion will be converted into a voltage, owing to the piezoelectric effect, achieving wave power extraction from ocean waves. Simultaneously, the motion of the waves will be damped. An analytical model was developed by Renzi (2016) to study the coupled hydroelectromechanical dynamics of a piezoelectric plate WEC consisting of a flexible bimorph plate submerged in the water with its ends clamped. The analytical study showed that the plate could extract a significant amount of energy at resonance, which might be used for low-power applications, 50 e.g., sensors and LEDs.

In addition to the water-wave interaction with submerged plates, wave scattering from floating elastic plates has also been widely studied due to its application in hydroelasticity of sea ice and very large floating structures (Squire, 2008). Sahoo et al. (2001) investigated the interaction of waves with a floating 55 semi-infinite elastic plate in finite water depth. Three edge conditions, i.e., a free edge, a simply supported edge, and a built-in edge, were considered in their study. The built-in edge condition resulted in the maximum wave reflection and the minimum wave transmission. Kohout et al. (2007) proposed an analytical model based on eigenfunction expansion method to study water wave propa- 
gation under a set of floating elastic plates occupying the entire water surface.

Regions of open water can be included as well, for the properties of the elastic plates can be set arbitrarily in their model. Praveen et al. (2018) investigated wave interaction with two floating elastic plates interconnected with vertical linear/rotational spring stiffness using the Timoshenko-Mindlin thick plate the65 ory. Analytical solutions for both finite and shallow water depths were derived. A significant reduction in the hydroelastic behaviour was observed due to the presence of articulation with varying spring stiffness. More recently, Koley et al. (2018) applied a Fredholm integral equation technique to study oblique wave scattering by a floating flexible porous plate. It was revealed that zero minima in wave reflection and full wave transmission occurred periodically with the increase in the plate length.

The above works deal with two-dimensional problems; three dimensional (3D) problems have received considerably less attention. Chwang and $\mathrm{Wu}$ (1994) applied linearized potential wave theory and the eigenfunction expan75 sion method to investigate the phenomenon of wave scattering by a submerged porous disk with an analytical model. Their analytical results indicated that an increase in the porous-effect parameter decreased the wave height. Another approach to the $3 \mathrm{D}$ wave scattering and radiation problems of a submerged disk based on a hypersingular integral equation was implemented by, e.g., Martin so and Farina (1997), Farina and Martin (1998) and Kundu et al. (2019). This method is difficult to generalise to problem which do not have circular symmetry. Meylan and Squire (1996) solved for a circular elastic plate floating in fluid of finite depth. Zilman and Miloh (2000) obtained a 3D, closed-form solution by using the angular eigenfunction expansion method for a circular thin elastic plate floating in shallow water. The circular geometry allowed the solutions for each angular eigenfunction to be decoupled from each other. Their method was later extended by Peter et al. (2004) to deal with the case of a circular elastic plate on water of finite depth. In order to account for the vertical variation of the spatial potential, vertical eigenfunctions were also employed in addition to 90 the angular eigenfunctions. For the plate-covered region two additional roots 
of the dispersion equation were chosen relative to those of the open-water region, and free-edge conditions - i.e., both the shear stress and bending moment vanish at the edge - were appended to close the system. $\mathrm{Xu}$ and $\mathrm{Lu}$ (2011) presented a further extension of the studies of Peter et al. (2004); Zilman and 95 Miloh (2000) to investigate the hydroelastic response of a plate with arbitrary shape floating on water of finite depth. Due to the non-circular geometry, the solutions for each angular eigenfunction were coupled with each other. In the expressions of the continuity conditions for pressure and velocity, the Fourier series expansion method was employed to deal with the terms associated with the radius function. The Fourier series expansion method for solving hydrodynamic problems of other marine structures with arbitrary cross-sections, e.g., wave radiation from a single floating truncated cylinder or multiple floating truncated cylinders, was applied by Yu et al. (2019); Zheng et al. (2019b). Montiel et al. (2013b a) carried out both analytical and experimental investigations on the hydroelastic response of two floating circular plates. Graf's addition theorem for Bessel functions (Kagemoto and Yue, 1986) was applied to consider the hydrodynamic interaction between the two plates. The front plate performed similarly to the single plate with additional small-amplitude peaks, which arise from the hydrodynamic interaction effect between the two plates. More recently, Mey110 lan et al. (2017) developed an analytical model to study water-wave scattering from a floating porous elastic circular plate. A quantity which is proportional to the energy dissipation induced by the porosity was also evaluated and it was concluded that there was an optimized porosity parameter to maximize the dissipation of wave energy by the plate.

In most of these studies concerning a floating elastic plate, the plate was assumed to be either non-porous, or circular, and generally, free-floating, i.e., the shear force and the bending moment vanish at the edge of the plate. However, in practice the plates may be neither non-porous nor circular for artificial structures, e.g., artificial floating islands (Kamble and Patil, 2012), floating vegetation fields (Duncan, 2014, Chen et al. 2016) and floating breakwaters (Cheng et al. 2013). Furthermore, their edges are generally fixed by piles, anchors, or 
tension cables. In such cases, the free-edge condition of the plates must be replaced by either the clamped edge condition or the simply supported edge condition.

In this paper, a semi-analytical model based on linear potential flow theory and an eigenfunction expansion method is developed to study wave scattering by a floating porous elastic plate with arbitrary shape. Three different types of edge condition, i.e., a clamped edge, a simply supported edge and a free edge, are considered. In particular, the wave power dissipated by the elastic plate due to porosity is evaluated.

The rest of this paper is organized as follows. The mathematical model for wave scattering is given in Section 2 The semi-analytical solutions of spatial velocity potentials can be found in Section 3. Section 4 presents methods for evaluating the scattered far-field amplitude function and the exact wave power dissipation. Validation and application of the semi-analytical model are given in Section 5. Finally, conclusions are drawn in Section 6 .

\section{Mathematical model for wave scattering}

In this paper, the scattering problem of a floating vegetated platform of arbitrary shape consisting of suspended vegetation is considered. Suspended vegetation is a porous medium, hence the platform can be simplified as a thin porous elastic plate of arbitrary shape (Fig. 11. The plate is located in water with a depth of $h$ and subjected to regular linear waves with small amplitude $A$. A Cartesian coordinate system $(x, y, z)$ is applied to describe the wave scattering problem with $O$ located at the centre of the plate, $z=0$ the mean water surface and $O z$ pointing upwards. Additionally, a local polar coordinate system $(r, \theta)$ is introduced in the plane of $z=0$ (Fig. 1 p). The incident wave direction $\beta$ is defined as the angle between the direction of wave propagation and the $O x$ axis.

The edge of the plate can be described in the polar coordinate system as a radius function $r=R(\theta)$, which represents the radius of any point at the 
(a)

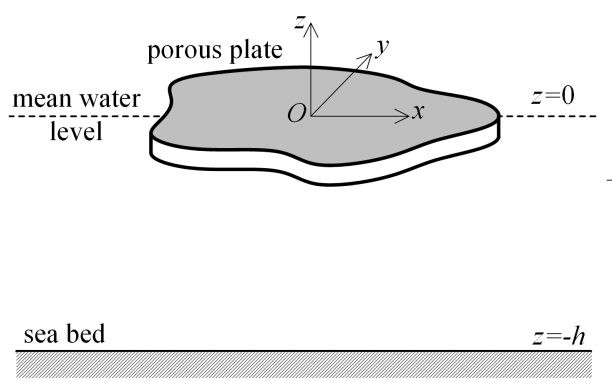

(b)

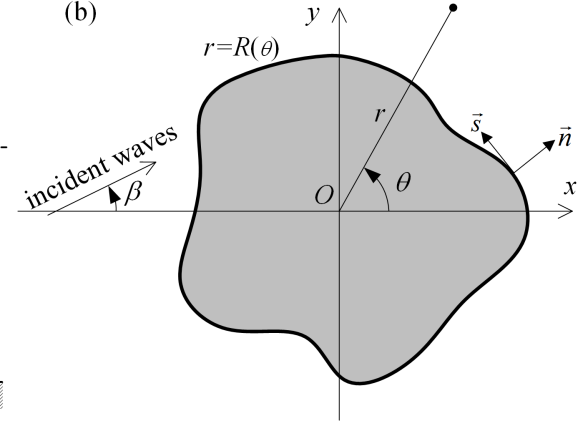

Figure 1: Schematic of the floating porous elastic plate of arbitrary shape: (a) side view; (b) plan view.

edge at $\theta$. Following Yu et al. (2019); Zheng et al. (2019b), the normal and tangential unit vectors pointing into the water at the edge in the polar system can be written respectively as:

$$
\vec{n}=\frac{1}{\sqrt{R^{2}+R^{\prime 2}}}\left(R \vec{e}_{r}-R^{\prime} \vec{e}_{\theta}\right), \quad \vec{s}=\frac{1}{\sqrt{R^{2}+R^{\prime 2}}}\left(R^{\prime} \vec{e}_{r}+R \vec{e}_{\theta}\right),
$$

where $\left(\vec{e}_{r}, \vec{e}_{\theta}\right)$ are the unit basis vectors in the polar coordinate system; $R^{\prime}$ denotes the derivative of $R$ with respect to $\theta$.

Assuming the water to be inviscid and incompressible, and considering linear potential flow, the velocity potential in the water domain may be written as $\operatorname{Re}\left[\Phi(x, y, z) \mathrm{e}^{-\mathrm{i} \omega t}\right]$, in which $\Phi$ is the spatial velocity potential to be determined, i denotes the imaginary unit, $\omega$ is the angular frequency of incident waves, and $t$ is time. $\Phi$ can be decomposed into two components,

$$
\Phi=\Phi_{\mathrm{I}}+\Phi_{\mathrm{S}}
$$

where $\Phi_{\mathrm{S}}$ represents the scattered velocity potential, and $\Phi_{\mathrm{I}}$ is the incident velocity potential, which may be expressed as (Zheng and Zhang, 2018, Zheng et al. 2018),

$$
\Phi_{\mathrm{I}}=-\frac{\mathrm{i} g A}{\omega} \frac{\cosh \left[k_{0}(z+h)\right]}{\cosh \left(k_{0} h\right)} \sum_{m=-\infty}^{\infty} \mathrm{i}^{m} \mathrm{e}^{-\mathrm{i} m \beta} J_{m}\left(k_{0} r\right) \mathrm{e}^{\mathrm{i} m \theta},
$$

where $g$ is the acceleration of gravity, $k_{0}$ is the wave number supporting propagating waves, and $J_{m}$ is the Bessel function of the $m$-th order. 
The velocity potential satisfies the Laplace equation in the whole water region,

$$
\left(\partial_{x}^{2}+\partial_{y}^{2}+\partial_{z}^{2}\right) \Phi=0
$$

and the impermeable condition at the sea bed,

$$
\partial_{z} \Phi=0, \quad z=-h .
$$

The whole water region can be divided into two domains, i.e., the exterior domain with $r>R$, and the interior domain with $r \leq R$. It is well known that both the kinematic and dynamic boundary conditions at the water surface of the exterior region should be satisfied, the combination of which gives

$$
-\omega^{2} \Phi+g \partial_{z} \Phi=0, \quad \text { for } \quad r>R, z=0 .
$$

The vertical displacement of the thin plate is defined as $\operatorname{Re}\left[\eta \mathrm{e}^{-\mathrm{i} \omega t}\right]$. Assuming that the lower surface of the plate remains in contact with the water all the time, $\Phi$ in the interior region can be coupled to the plate displacement function, $\eta$, in terms of both kinematic and dynamic conditions (Meylan, 2002),

$$
\partial_{z} \Phi=-\mathrm{i} \omega \eta+\mathrm{i} P \Phi, \quad g\left[\chi \Delta^{2}+1-\left(\omega^{2} / g\right) \gamma\right] \eta-\mathrm{i} \omega \Phi=0, \quad \text { for } r \leq R, z=0,
$$

where $P$ represents the porosity parameter, which may be derived from Darcy's law based on the assumption that the constant atmospheric pressure is normalized to zero, $P=\omega K \rho /(\mu h)$, in which $\rho$ and $\mu$ denote the density and dynamic viscosity of water, respectively, and $K$ the permeability of the plate. $\gamma$ and $\chi$ denote the mass per unit area and the flexural rigidity of the plate, respectively, scaled with respect to $\rho . \Delta$ is the Laplacian operator in the horizontal plane, which can be expressed as $\Delta=\partial_{x}^{2}+\partial_{y}^{2}$ in the Cartesian coordinate system and $\Delta=\partial_{r}^{2}+r^{-1} \partial_{r}+r^{-2} \partial_{\theta}^{2}$ in the polar system, respectively.

The two components as given in Eq. (7) can be combined into

$$
\left(\omega^{2} / g\right) \Phi=\left[\chi \partial_{z}^{4}+1-\left(\omega^{2} / g\right) \gamma\right]\left(\partial_{z}-\mathrm{i} P\right) \Phi, \quad \text { for } r \leq R, z=0 .
$$

The scattered spatial potential $\Phi_{\mathrm{S}}$ should also satisfy the Sommerfeld radiation condition in the far field. Additionally, the edge boundary conditions of 
the thin elastic plate should be taken into account. In this paper, three different edge conditions of the plate are considered, i.e., a clamped edge, a simply supported edge and a free edge.

For a clamped edge, the plate displacement and rotation at the edge are restrained, producing zero deflection and slope there, i.e.,

$$
\eta=0, \quad \partial_{n} \eta=0, \quad \text { for } r=R,
$$

in which $\partial_{n}$ represents the differential operator corresponding to the normal vector.

For a simply supported edge, the plate displacement on the edge is restrained, whereas the plate is free to rotate, i.e., the bending moment, $M$, vanishes, which yields the condition:

$$
M=0, \quad \eta=0, \quad \text { for } r=R .
$$

For a free edge, the plate is unrestrained on the edge, i.e., both the bending moment, $M$, and the shear stress, $V$, vanish, providing the condition:

$$
M=0, \quad V=0, \quad \text { for } r=R .
$$

Expressions of the bending moment, $M$, and the shear stress, $V$, on the edge of the plate in the polar system can be derived based on Reddy (2007); Timoshenko and Woinowsky-Krieger (1959), and their final expressions are given in Appendix A

\section{Analytical solution of spatial velocity potentials}

An expression for the velocity potential in the exterior domain, i.e., $r>R$, can be written as

$$
\Phi_{\mathrm{ext}}(r, \theta, z)=\Phi_{\mathrm{I}}+\sum_{m=-\infty}^{\infty} \sum_{l=0}^{\infty} A_{m, l} H_{m}\left(k_{l} r\right) Z_{l}(z) \mathrm{e}^{\mathrm{i} m \theta}
$$

where $A_{m, l}$ are the unknown coefficients to be determined. $H_{m}$ represents the Hankel function of the $m$-th order; $Z_{l}(z)=\frac{\cosh \left[k_{l}(z+h)\right]}{\cosh \left(k_{l} h\right)}$, in which $k_{0} \in \mathbb{R}^{+}$ 
and $k_{l} \in \mathrm{i} \mathbb{R}^{+}$for $l=1,2,3, \ldots$ support the propagating waves and evanescent waves, respectively, and they are the positive real root and the infinite positive imaginary roots of the dispersion relation derived from Eq. (6) for the exterior region (Zheng et al. 2018, Zheng and Zhang, 2018, Zheng et al., 2019a),

$$
\omega^{2}=g k_{l} \tanh \left(k_{l} h\right)
$$

The velocity potential in the interior domain, i.e., $r \leq R$, can be expressed as

$$
\Phi_{\text {int }}(r, \theta, z)=\sum_{m=-\infty}^{\infty} \sum_{l=-2}^{\infty} B_{m, l} J_{m}\left(\kappa_{l} r\right) Y_{l}(z) \mathrm{e}^{\mathrm{i} m \theta}
$$

where $B_{m, l}$ are the unknown coefficients to be determined; $Y_{l}(z)=\frac{\cosh \left[\kappa_{l}(z+h)\right]}{\cosh \left(\kappa_{l} h\right)}$, in which $\kappa_{l}(l=-2,-1,0,1,2,3, \ldots)$ are the roots of the dispersion relation derived from Eq. (8) for the interior region (Meylan et al., 2017),

$$
\left[\chi \kappa_{l}^{4}+1-\left(\omega^{2} / g\right) \gamma\right]\left[\kappa_{l} \tanh \left(\kappa_{l} h\right)-\mathrm{i} P\right]=\omega^{2} / g
$$

For $P=0, \kappa_{0} \in \mathbb{R}^{+}$and $\kappa_{l} \in \mathrm{i} \mathbb{R}^{+}$for $l=1,2,3, \ldots$ can be obtained, which support the propagating waves and evanescent waves, respectively, similarly to the exterior region. The remaining two roots, $\kappa_{-2}$ and $\kappa_{-1}$, support damped propagating waves, and satisfy $\kappa_{-1} \in \mathbb{R}^{+}+\mathrm{i} \mathbb{R}^{+}$and $\kappa_{-2}=-\kappa_{-1}^{*}$, in which * denotes the complex conjugate. The $\kappa_{l}$ for $l=0,1,2,3, \ldots$ and $k_{l}$ for $l=$ $1,2,3, \ldots$ can be calculated using a bisection method. The two complex roots $\kappa_{-2}$ and $\kappa_{-1}$ can be determined by means of Muller's method (Press et al. 2007).

When $P \neq 0$, the structure of $\kappa_{l}$ is perturbed (Meylan et al., 2017). Generally, neither pure real nor pure imaginary roots exist, and the symmetry between $\kappa_{-2}$ and $\kappa_{-1}$ is not valid either. A homotopy method is employed to calculate the roots of Eq. 13 with $P \neq 0$, starting with the corresponding roots for the case of $P=0$ and connecting them with the roots to be determined for $P \neq 0$ by incrementing $P$ from 0 to the specified value (Meylan et al. 2017). Eq. (13) may be nondimensionalised,

$$
\left[\bar{\chi} \bar{\kappa}_{l}^{4}+1-h\left(\omega^{2} / g\right) \bar{\gamma}\right]\left[\bar{\kappa}_{l} \tanh \left(\bar{\kappa}_{l}\right)-\mathrm{i} \bar{P}\right]=h \omega^{2} / g
$$




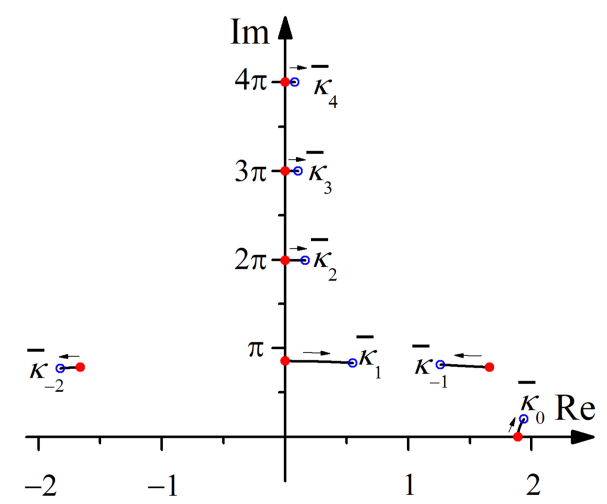

Figure 2: Loci of the nondimensional roots of the dispersion relation for the interior region, i.e., Eq. (14), for $l=-2, \ldots, 4$, in the complex plane with $h \omega^{2} / g=2.0, \bar{\chi}=\bar{\gamma}=0.01$, where the red solid points and blue circles correspond to the roots $\bar{\kappa}_{l}$ for $\bar{P}=0$ and 1.0, respectively.

in which $\bar{\chi}=\chi / h^{4}, \bar{\gamma}=\gamma / h, \bar{\kappa}_{l}=\kappa_{l} h$ and $\bar{P}=P h$. Taking $h \omega^{2} / g=2.0, \bar{\chi}=$ $\bar{\gamma}=0.01$, as an example, the $\bar{\kappa}_{l}$ for $l=-2,-1,0,1,2,3$ and 4 corresponding to $\bar{P}=0$ and 1.0 are plotted in the complex plane in Fig. 2, in which the lines represent the path of the homotopy as $\bar{P}$ is varied from 0 to 1.0.

Using the first component of Eq. (7) and Eq. (12), the plate displacement function, $\eta$, may be expressed in terms of the unknown coefficients $B_{m, l}$ :

$$
\eta=\frac{\mathrm{i} \omega}{g} \sum_{m=-\infty}^{\infty} \sum_{l=-2}^{\infty} \frac{B_{m, l} J_{m}\left(\kappa_{l} r\right)}{\chi \kappa_{l}^{4}+1-\left(\omega^{2} / g\right) \gamma} \mathrm{e}^{\mathrm{i} m \theta}=0 .
$$

The velocity potentials in the exterior and interior domains, Eqs. 10 and (12), must satisfy the continuity conditions:

(1) Continuity of pressure at the boundary $r=R$,

$$
\left.\Phi_{\mathrm{int}}\right|_{r=R}=\left.\Phi_{\mathrm{ext}}\right|_{r=R}, \quad-h<z<0,
$$

(2) Continuity of normal velocity at the boundary $r=R$,

$$
\left.\left(R^{2} \frac{\partial \Phi_{\mathrm{int}}}{\partial r}-R^{\prime} \frac{\partial \Phi_{\mathrm{int}}}{\partial \theta}\right)\right|_{r=R}=\left.\left(R^{2} \frac{\partial \Phi_{\mathrm{ext}}}{\partial r}-R^{\prime} \frac{\partial \Phi_{\mathrm{ext}}}{\partial \theta}\right)\right|_{r=R}, \quad-h<z<0 .
$$

After inserting the expression of the spatial potentials into the continuity conditions and expanding the functions relating to the radius function of the 
plate edge into a Fourier series, the wave scattering problem can be solved with an eigenfunction-matching method. Detail derivation and calculation for the unknown coefficients are given in Appendix B.

\section{Scattered far-field amplitude function and power dissipation}

The terms pertaining to the evanescent wave components in the scattered velocity potential decay exponentially with increasing $r$. Hence only the propagating wave components remain for large values of $k r$, where $k$ is employed to represent $k_{0}$ for simplification. With the asymptotic forms of $H_{m}$ for $k r \rightarrow \infty$,

$$
H_{m}(k r)=\sqrt{2 / \pi} \mathrm{e}^{-\mathrm{i}(m \pi / 2+\pi / 4)}(k r)^{-1 / 2} \mathrm{e}^{\mathrm{i} k r}, \quad k r \rightarrow \infty,
$$

the scattered wave elevation, $\eta_{\mathrm{R}}$, in the far field can be evaluated by

$$
\eta_{\mathrm{R}}=\frac{\mathrm{i} \omega}{g} \Phi_{\mathrm{S}}=\sqrt{2 \pi} \sigma_{\mathrm{R}}(\theta)(k r)^{-1 / 2} \mathrm{e}^{\mathrm{i}(k r-\pi / 4)}, \quad k r \rightarrow \infty,
$$

in which $\sigma_{\mathrm{R}}$ is the so-called far-field amplitude function (Meylan et al., 2017)

$$
\sigma_{\mathrm{R}}(\theta)=\frac{\mathrm{i} \omega}{g \pi} \sum_{m=-\infty}^{\infty} A_{m, 0} \mathrm{e}^{\mathrm{i} m(\theta-\pi / 2)}
$$

Unlike Meylan et al. (2017), who calculated a quantity (denoted as $E$ ) that is proportional to the wave energy dissipated due to the porosity by integrating the far-field amplitude functions, here we employ a straightforward way to evaluate the exact wave power dissipation,

$$
\begin{aligned}
P_{\text {diss }} & =\frac{P}{2 \rho \omega} \iint_{\Omega}|p|^{2} \mathrm{~d} s=\frac{\rho \omega P}{2} \iint_{\Omega}\left|\Phi_{\text {int }}(r, \theta, 0)\right|^{2} \mathrm{~d} s \\
& =\frac{\rho \omega P}{2} \iint_{\Omega}\left|\sum_{m=-\infty}^{\infty} \sum_{l=-2}^{\infty} B_{m, l} J_{m}\left(\kappa_{l} r\right) \mathrm{e}^{\mathrm{i} m \theta}\right|^{2} \mathrm{~d} s,
\end{aligned}
$$

where $\Omega$ represents the plate area, and $p=\mathrm{i} \omega \rho \Phi_{\text {int }}$ denotes the hydrodynamic pressure under the plate.

The wave power dissipation may be nondimensionalized,

$$
\eta_{\text {diss }}=k P_{\text {diss }} / P_{\text {in }}
$$

with $P_{\text {in }}$ the incident wave power per unit width of wave front,

$$
P_{\text {in }}=\frac{\rho g A^{2}}{2} \frac{\omega}{2 k}\left[1+\frac{2 k h}{\sinh (2 k h)}\right] \text {. }
$$




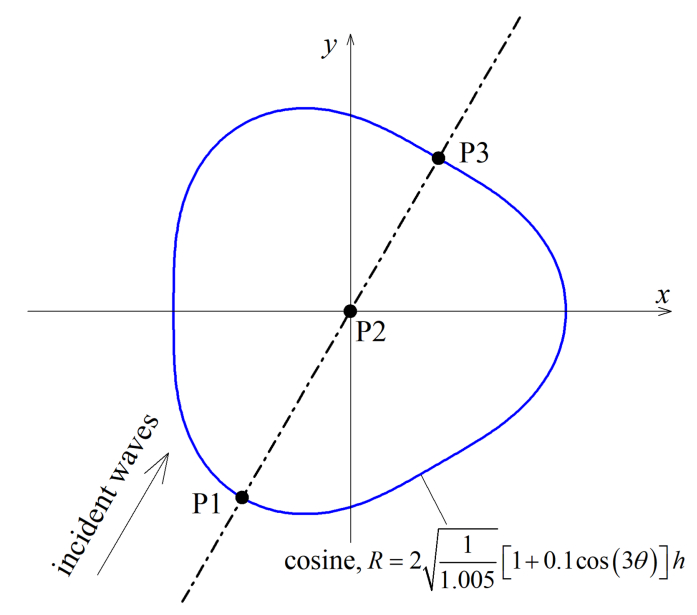

Figure 3: Schematic of a cosine-shaped plate (plan view).

\section{Results}

\subsection{Convergence analysis}

A cosine-shaped free floating porous elastic plate in incident regular waves with wave direction $\beta=\pi / 3$ (Fig. 3 ) is taken as a case study to examine the numerical performance of the model. P1, P2 and P3 are three selected points along a line of symmetry of the cosine-shaped plate to demonstrate the plate response.

Figures 4 and 5 illustrate the impact of the angular and vertical truncated terms (i.e., in terms of $M$ and $L$ ), respectively, on variation of the deflection amplitudes at P1, P2 and P3 of the porous elastic plate with $\bar{P}$ for $k h=2.0$, $\bar{\chi}=\bar{\gamma}=0.01$. Additionally, the CPU time requirements, together with the results of the deflection amplitudes at P1, P2 and P3, for different number of truncated terms for the plate with $\bar{\chi}=\bar{\gamma}=0.01, k h=2.0$ and $\bar{P}=1.0$ are plotted in Fig. 6 .

In order to obtain the converged results, $M \geq 9$ and $L \geq 3$ are suggested. It can be seen that the CPU time increases sharply when increasing the number of angular or vertical truncated terms (Fig. 6). Hereinafter, $M=12$ and $L=4$ are adopted to achieve convergence at a low computational cost. 

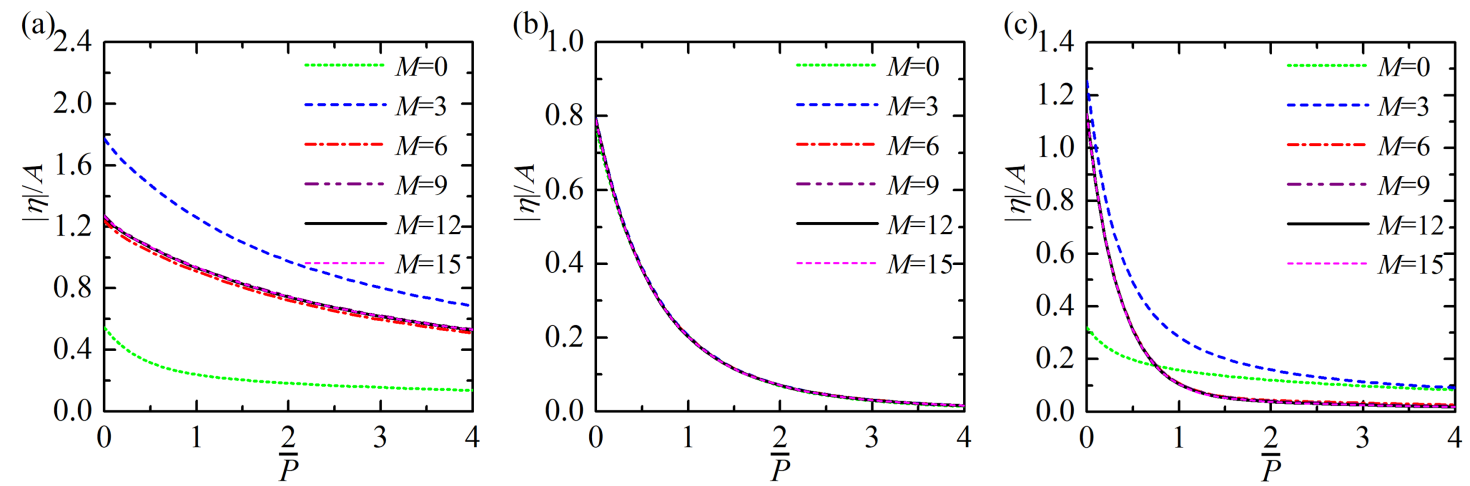

Figure 4: Impact of the angular cutoffs (i.e., in terms of $M$ ) on the deflection amplitude of a cosine-shaped porous elastic plate, $k h=2.0, \bar{\chi}=\bar{\gamma}=0.01$ and $L=4$ : (a) $|\eta| / A$ at P1; (b) $|\eta| / A$ at $\mathrm{P} 2 ;$ (c) $|\eta| / A$ at $\mathrm{P} 3$.
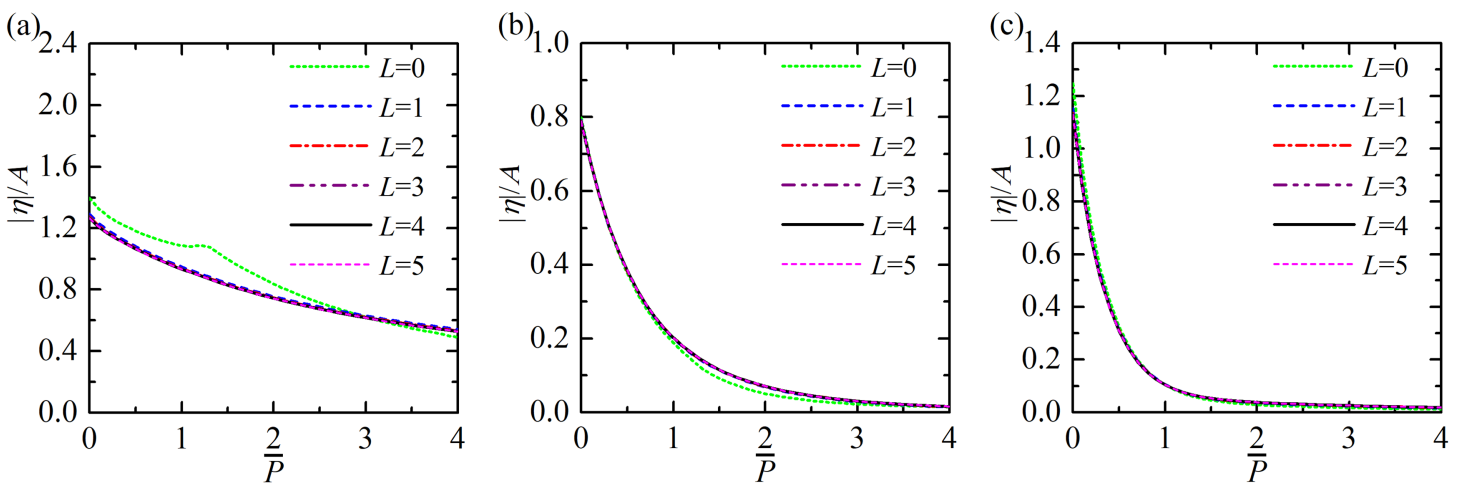

Figure 5: Impact of the vertical cutoffs (i.e., in terms of $L$ ) on the deflection amplitude of a cosine-shaped porous elastic plate, $k h=2.0, \bar{\chi}=\bar{\gamma}=0.01$ and $M=12:(\mathrm{a})|\eta| / A$ at P1; (b) $|\eta| / A$ at $\mathrm{P} 2 ;(\mathrm{c})|\eta| / A$ at $\mathrm{P} 3$. 

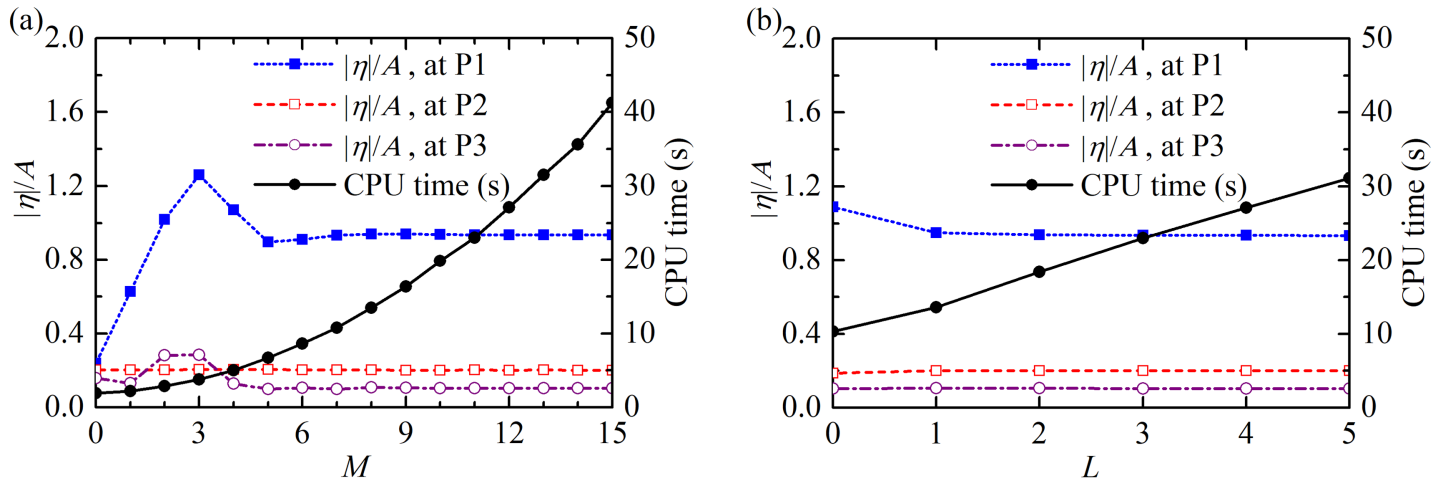

Figure 6: Variation of $|\eta| / A$ at P1, P2, P3, and CPU time required with the number of cutoffs for $k h=2.0, \bar{\chi}=\bar{\gamma}=0.01$ and $\bar{P}=1.0$ : (a) angular cutoffs (i.e., in terms of $M$ ), $L=4$; (b) vertical cutoffs (i.e., in terms of $L$ ), $M=12$.

\subsection{Validation of the semi-analytical model}

The elliptical floating non-porous elastic plate simulated by $\mathrm{Xu}$ and $\mathrm{Lu}$ (2011) is adopted here as a case study to validate the present model. The radius function of the elliptical plate can be written as

$$
R(\theta)=\left[\frac{\cos ^{2}(\theta+\vartheta)}{a^{2}}+\frac{\sin ^{2}(\theta+\vartheta)}{b^{2}}\right]^{-1 / 2},
$$

in which $a$ and $b$ represent the semi-major and semi-minor axes of the plate, respectively; and $\vartheta$ denotes the angle between the major axis and the negative $x$-axis.

Deflection amplitudes of the elliptical plate with a clamped edge and a simply supported edge are plotted in Figs. 7 and 8 respectively. Excellent agreement with the results of $\mathrm{Xu}$ and $\mathrm{Lu}(2011)$ is obtained.

In addition to the non-porous plate, a circular porous elastic plate previously investigated by Meylan et al. (2017) is selected as the second case to validate the present model. Figure 9 illustrates the instantaneous deflection of the plate with a free edge at $t=0$ with different values of the porosity parameter $\bar{P}$. Additionally, the scattered far-field amplitude function of the plate for $\bar{P}=0.5$ and $\beta=\pi / 6$ is given in Fig. 10a. The energy dissipated due to porosity as a function of $\bar{P}$ is provided in Fig. 10b. The present results of the plate 

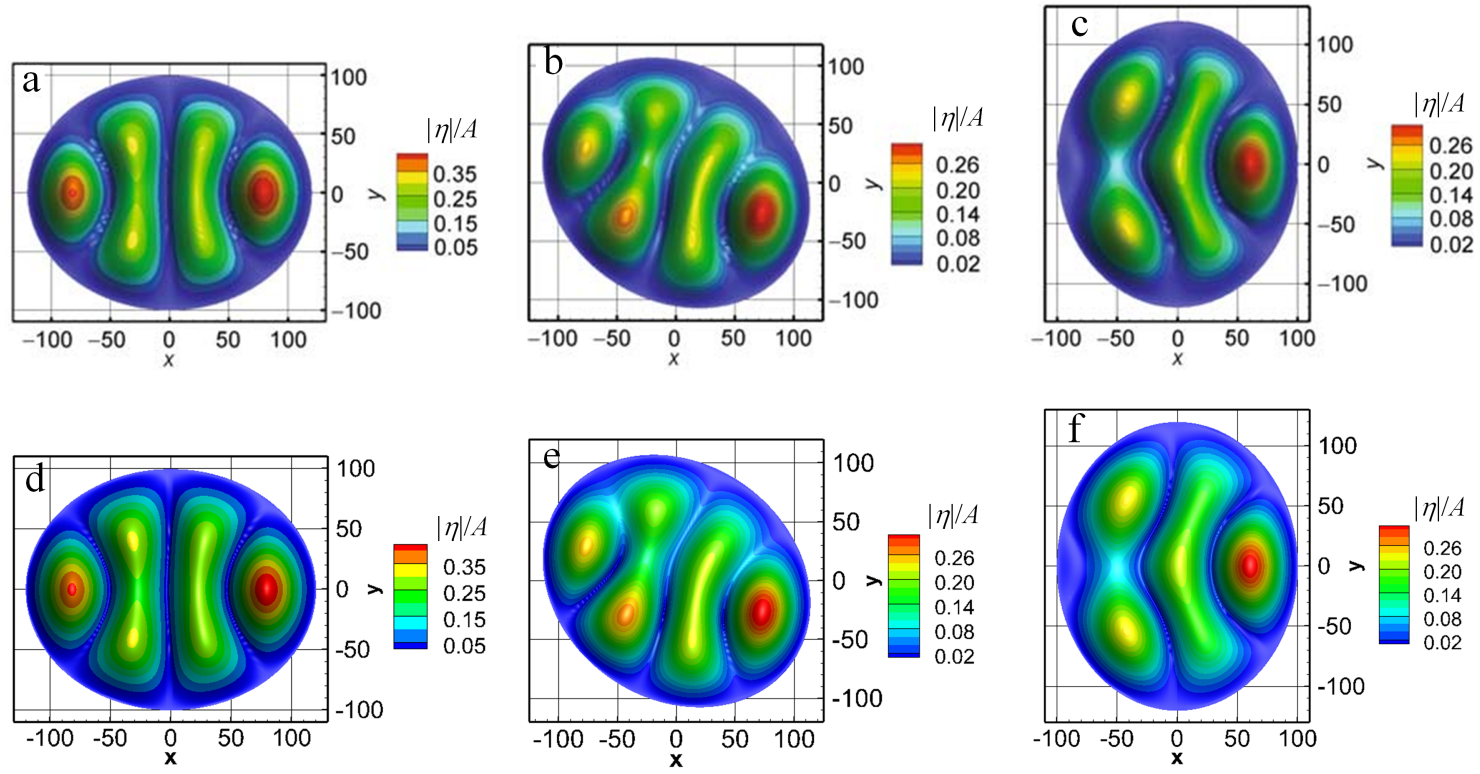

Figure 7: Deflection amplitudes of an elliptic plate with a clamped edge for different deployment (from left to right, $\vartheta=0, \pi / 5$ and $\pi / 2$ ) with the semi-major axis $a=120 \mathrm{~m}$, semi-minor axis $b=100 \mathrm{~m}, \beta=0, h=25 \mathrm{~m}, k h=\pi, \chi=10^{5} \mathrm{~m}^{4}$ and $\gamma=P=0$. (a)-(c) Results of Xu and $\mathrm{Lu}(2011)$ and (d)-(f) present results. 

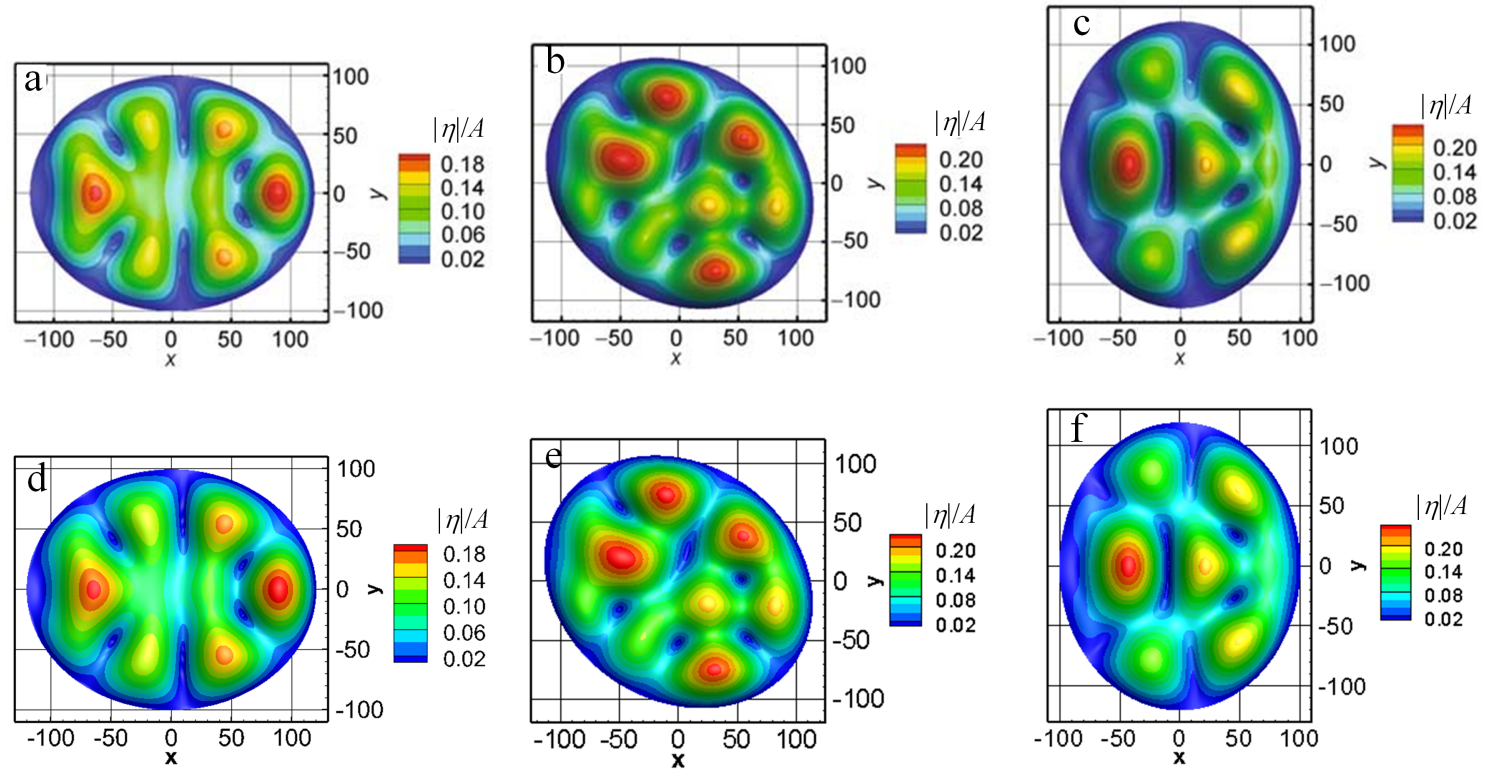

Figure 8: Deflection amplitudes of an elliptic plate with a simply supported edge for different deployment (from left to right, $\vartheta=0, \pi / 5$ and $\pi / 2$ ) with the semi-major axis a $=120 \mathrm{~m}$, semi-minor axis $b=100 \mathrm{~m}, \beta=0, h=25 \mathrm{~m}, k h=\pi, \chi=10^{5} \mathrm{~m}^{4}$ and $\gamma=P=0$. (a)-(c) Results of $\mathrm{Xu}$ and $\mathrm{Lu}(2011$ and (d)-(f) present results. 

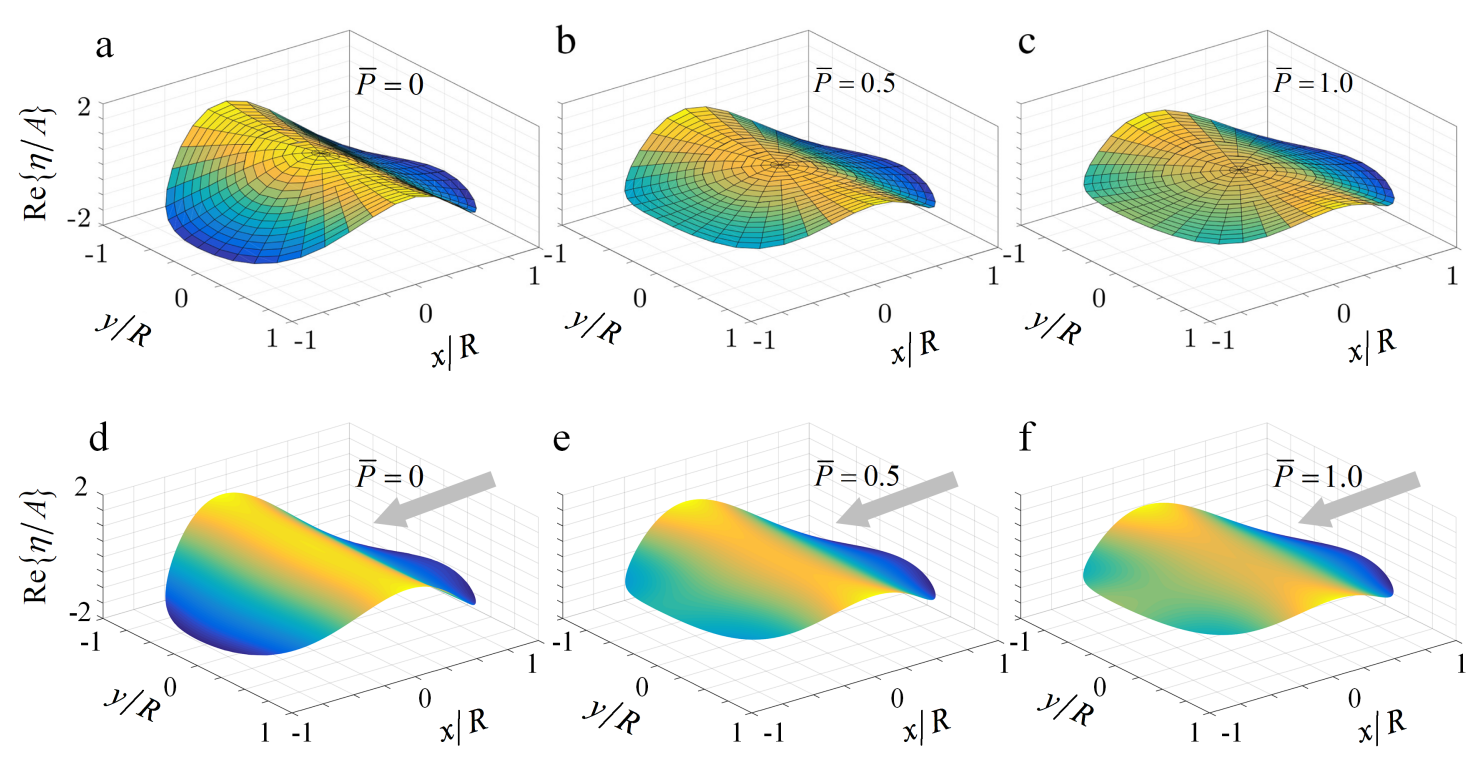

Figure 9: Deflection of a circular plate with a free edge at $t=0$ with different values of $\bar{P}$ for $R / h=2, \beta=\pi, h \omega^{2} / g=2.0$ and $\bar{\chi}=\bar{\gamma}=0.01$. (a)-(c) Results of Meylan et al. (2017), where a typo of the incident wave direction existed (i.e., $\beta$ was typed as 0 rather than $\pi$ ) and (d)-(f) present results.

deflection and the scattered far-field amplitude function are found to be in excellent agreement with the published results (Meylan et al., 2017). The trend of the exact dissipated power versus the porosity parameter coincides well with that of the quantity proportional to the dissipated power (Fig. 10b).

The excellent agreement (Figs. 7 10 gives confidence in the present semianalytical model for solving wave scattering by a floating porous elastic plate of arbitrary shape.

\subsection{Performance of a floating porous elastic plate with different shapes}

In this section, three floating porous elastic plates with different shapes and pdge conditions are considered to represent floating vegetated platforms (Duncan, 2014, Chen et al. 2016) composed of suspended vegetation with different layout, which are employed to dissipate waves. Figure 11 presents a top view of the plates with different shapes, i.e., circular, cosine and elliptical shapes. The 

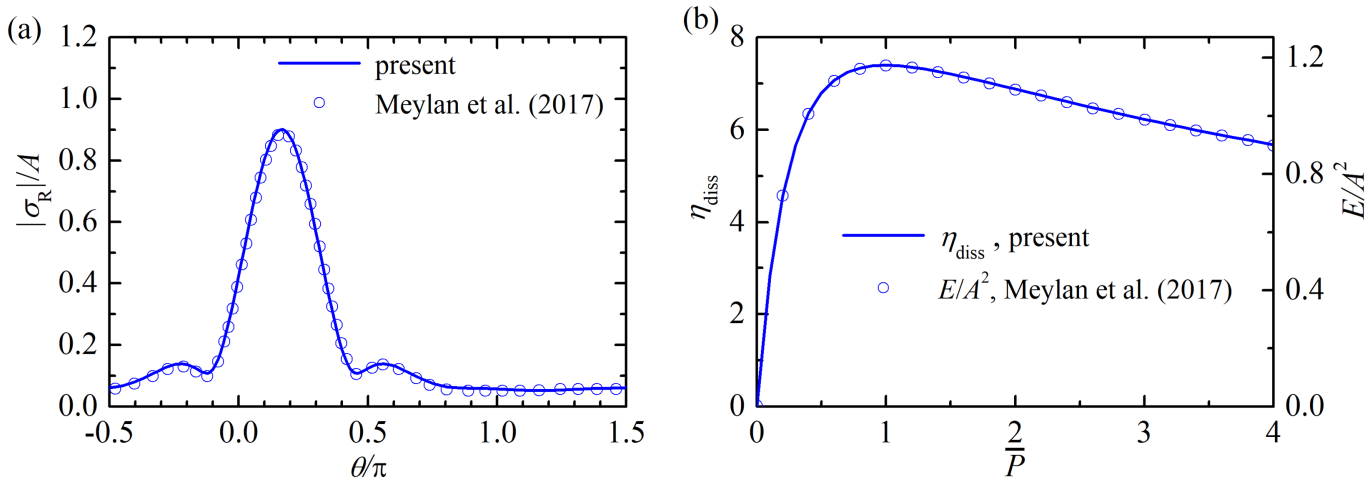

Figure 10: (a) Scattered far-field amplitude function of a circular plate with a free edge for $\beta=\pi / 6, \bar{P}=0.5$, and (b) nondimensional wave power dissipation by a circular plate with a free edge versus the porosity parameter, $\bar{P}$. $\left[R / h=2, h \omega^{2} / g=2.0, \bar{\chi}=\bar{\gamma}=0.01\right]$.

radius function $R(\theta)$ corresponding to each plate shape is provided in Fig. 11 as well, which ensures that all these plates have the same area, i.e., $4 \pi h^{2}$. Since the performance of the plate with cosine or elliptical shape may be dependent on the incident wave direction, five cases are examined by applying the present validated semi-analytical model: case 1 , circular plate with $\beta=0$; case $2 \mathrm{a}$, cosine plate with $\beta=0$; case $2 \mathrm{~b}$, cosine plate with $\beta=\pi / 3$; case $3 \mathrm{a}$, elliptical plate with $\beta=0$; case $3 \mathrm{~b}$, elliptical plate with $\beta=\pi / 2$.

\subsubsection{Performance of the plate with a free edge}

Figure 12 presents the variation of the maximum deflection amplitude, $|\eta|_{\max } / A$, and the nondimensional dissipated power, $\eta_{\text {diss }}$, of the plate with a free edge in different cases versus the porosity.

As expected, the maximum deflection amplitude of each plate as plotted in Fig. 12 a shows a monotonous decrease as the porosity becomes larger. The curves of $|\eta|_{\max } / A$ versus $\bar{P}$ for the five cases nearly overlap each other, except for $\bar{P}<0.5$. When $\bar{P}=0$, i.e., the plate is non-porous, $|\eta|_{\max } / A$ is around 1.25 , and in particular, it reaches the maximum value of 1.30 for case $3 \mathrm{~b}$. As a comparison, $|\eta|_{\max } / A$ is reduced to 0.52 when $\bar{P}=4.0$ for all the cases examined, indicating the effectiveness of the porosity in damping the plate response. 


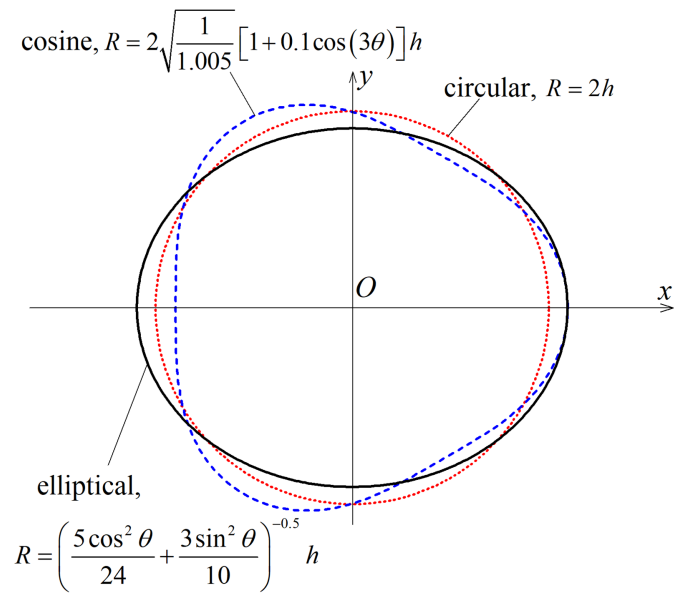

Figure 11: Schematic of the three plates with different shapes.

As indicated in Fig. $12 \mathrm{~b}$, no wave power can be dissipated by the plate when $\bar{P}=0$, i.e., the plate is non-porous. As $\bar{P}=0$ increases from 0 towards $4.0, \eta_{\text {diss }}$ first increases from 0 , and then decreases after reaching a peak around $\bar{P}=1.0$, regardless of the plate shapes. This is reasonable because when $\bar{P}$ is too large, the pressure under the plate and the plate response might be too small to dissipate wave power. The peak value of $\eta_{\text {diss }}$ and the corresponding $\bar{P}$ for the five cases are $(7.20,1.0),(7.67,1.1),(6.91,0.9),(6.65,0.9)$ and $(7.83$, 1.0), respectively. For most values of the porosity, especially in the range of $0.5<\bar{P}<1.5$, the plate in case 3b, i.e., the elliptical plate with its major axis perpendicular to the incident wave direction, works better than the others in power dissipation. The least power dissipation is found for the plate in case 3a, i.e., the elliptical plate, whereas with its minor axis perpendicular to the incident wave direction. The second best plate is the one in case 2a, i.e., the cosine plate with its flat edge facing the incident waves. This plate works even better than the elliptical plate in case $3 \mathrm{~b}$ for a large porosity, e.g., $\bar{P}>2.5$. When the sharp edge of the cosine plate faces to the incident waves (i.e., case $2 \mathrm{~b}$ ), a larger value of $\eta_{\text {diss }}$ is obtained compared to that of the plate in case 3 a for the whole computed range of $\bar{P}$. Although the circular plate holds an intermediate ability 

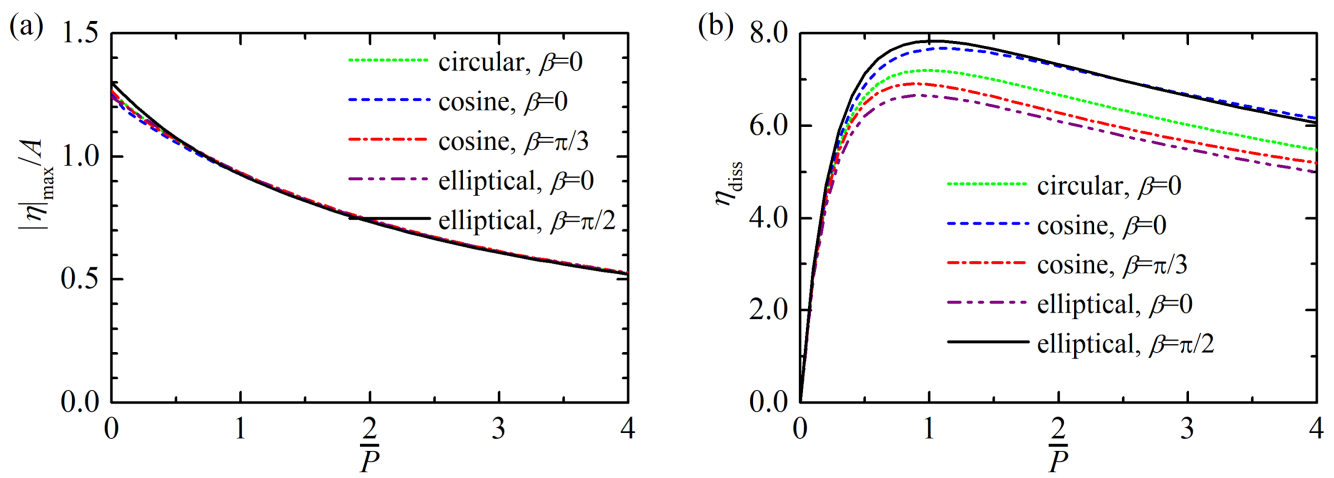

Figure 12: (a) Maximum deflection amplitude (i.e., $|\eta| \max / A$ ) and (b) Power dissipation (i.e., $\left.\eta_{\text {diss }}\right)$ of the plates with a free edge as functions of the porosity parameter, $\bar{P}$. $[k h=2.0$, $\bar{\chi}=\bar{\gamma}=0.01]$.

in power dissipation, as shown in Fig. 12 , it may be advantageous due to its constant performance independent of incident wave direction.

The deflection of the plate with a free edge in the five cases in terms of deflection amplitude and the instantaneous deflection at $t=0$ for $\bar{P}=0$ (i.e., non-porous) and 1.0, are plotted in Figs. 13 and 14 respectively. For all the five cases with $\bar{P}=0$ or 1.0 , the deflection is symmetrical with respect to the symmetrical plate axis along the incident propagating direction.

For a non-porous plate, the deflection at both the waveward edge and the opposite edge is larger than those for other regions of the plate (Fig. 13). There are two separate internal regions of the plate where the response is weaker than the other regions. The smallest deflection occurs at the leeward internal region. For $\bar{P}=1.0$, the strong response of the plate merely occurs at the front edge (Fig. 14). The two internal regions where the weak deflection occurs in a nonporous plate merge into one for $\bar{P}=1.0$, with its position moving towards the leeward edge. This can be explained by the power dissipation due to the porosity, which results in less power propagating towards the leeward edge, and hence a weaker wave motion and plate response. As may be inferred from the comparison between Figs. 13 and 14 the overall deflection of the plate in all 

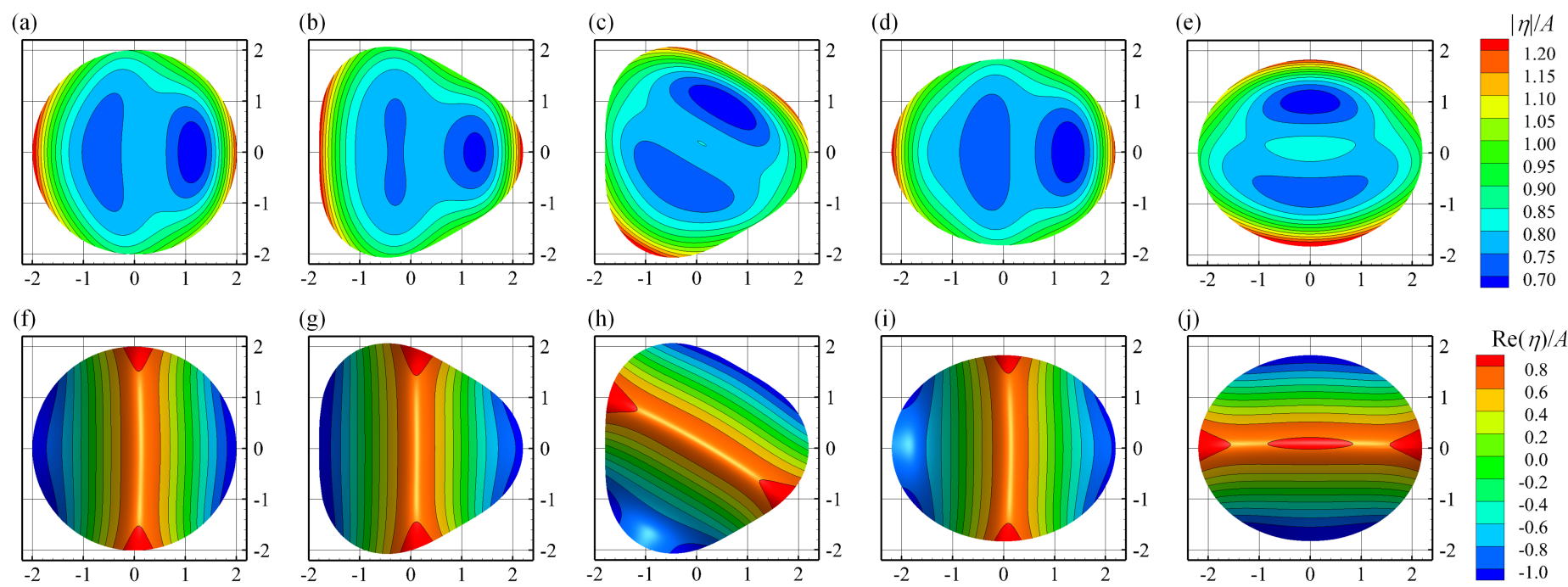

Figure 13: Deflection of the plate with a free edge in different cases for $\bar{P}=0$, (a)-(e) deflection amplitude, $|\eta| / A$, and (f)-(j) instantaneous deflection at $t=0, \operatorname{Re}(\eta) / A$. $[k h=2.0$, $\bar{\chi}=\bar{\gamma}=0.01]$.

the five cases weakens as $\bar{P}$ increases from 0 to 1.0 .

\subsubsection{Performance of the plate with a simply supported edge}

The variation of the maximum deflection amplitude and the dissipated power of the plate with a simply supported edge in different cases versus the porosity parameter is plotted in Fig. 15.

As shown in Fig. 15a, the maximum deflection amplitude $\left(|\eta|_{\max } / A\right)$ of each plate with a simply supported edge is observed to decrease with the increase of the porosity parameter $(\bar{P})$. This decrease is, however, different from that for the plate with a free edge (Fig. 12 ), where the declining trend of $|\eta|_{\max } / A$ versus $\bar{P}$ is steady across the entire range of $\bar{P}$ considered. The variation of $|\eta|_{\max } / A$ for a simply supported edge (Fig. 15) with $\bar{P}$ can be divided into two stages: stage one, $\bar{P}<0.5$, where $|\eta|_{\max } / A$ is rather sensitive to the change of $\bar{P}$; stage two, $\bar{P}>0.5$, for which as $\bar{P}$ keeps increasing towards 4.0 , the changing rate of $|\eta|_{\max } / A$ with $\bar{P}$ is obviously smaller than that in the first stage. Taking case $3 \mathrm{~b}$ (i.e., elliptical plate with $\beta=\pi / 2$ ) as an example, $|\eta|_{\max } / A$ drops dramatically 
(a)

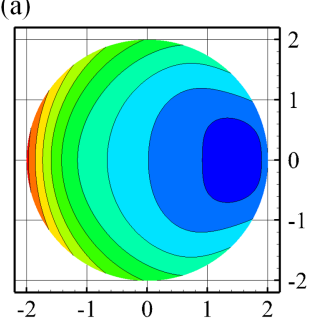

(f)

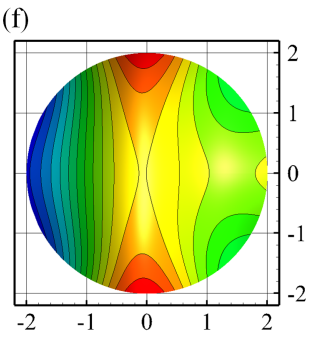

(b)

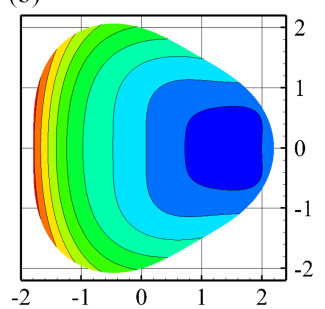

(g)

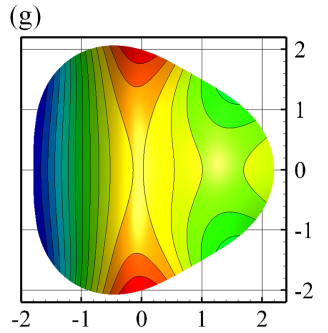

(c)

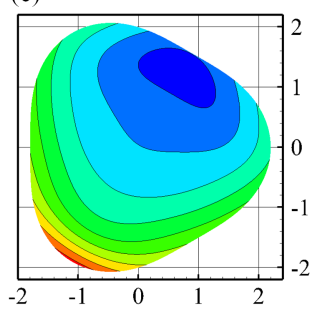

(h)

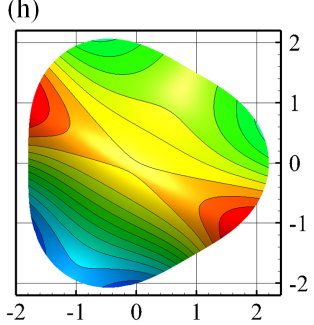

(d)

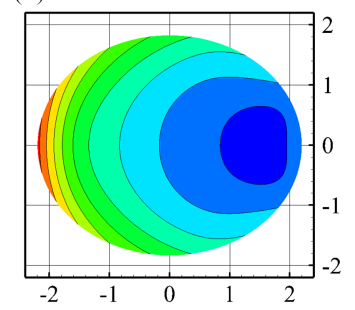

(i)

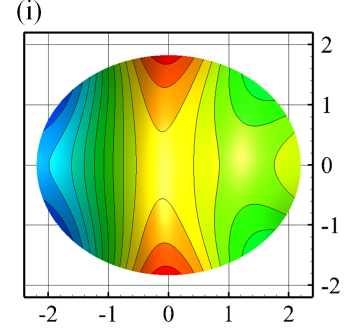

(e)

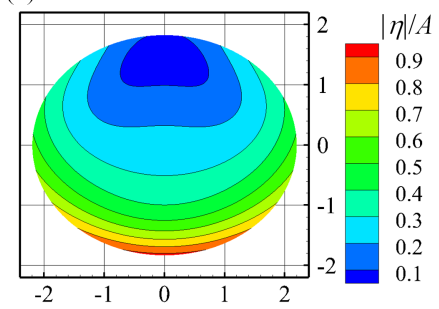

(j)

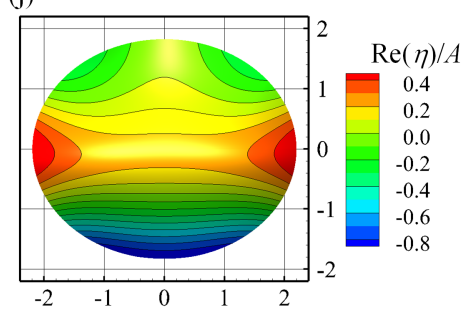

Figure 14: Deflection of the plate with a free edge in different cases for $\bar{P}=1.0$, (a)-(e) deflection amplitude, $|\eta| / A$, and (f)-(j) instantaneous deflection at $t=0, \operatorname{Re}(\eta) / A$. $[k h=2.0$, $\bar{\chi}=\bar{\gamma}=0.01]$.

from 1.41 to 0.56 as $\bar{P}$ increases from 0 to 0.5 , and $|\eta|_{\max } / A=0.16$ is ultimately obtained at $\bar{P}=4.0$. For $\bar{P}<1.0$, different values of $|\eta|_{\max } / A$ can be achieved for different cases and the largest one among the five cases examined occurs in case 3 b. For $\bar{P}>1.0,|\eta|_{\max } / A$ of the plate is nearly independent of the plate shape.

Compared to the results for the free edge conditions (Fig. 12b), the peak of the $\eta_{\text {diss }}-\bar{P}$ curve for the simply supported edge as plotted in Fig. $15 \mathrm{~b}$ is much flatter and it occurs at a larger value of porosity $(\bar{P} \approx 1.6)$. A relatively steady level of $\eta_{\text {diss }}$ is observed with $\bar{P}$ increasing from 1.0 to 4.0. In the computed range of $\bar{P}$, the overall wave power dissipated by the plate with a simply supported edge is obviously smaller than that dissipated by the plate with a free edge. Comparison of the performance of the plate with a simply supported edge in the five examined cases in terms of wave power dissipation looks similar to that for the free edge conditions (Section 5.3.1).

Figs. 16 and 17 present the deflection of the plate with a simply supported 

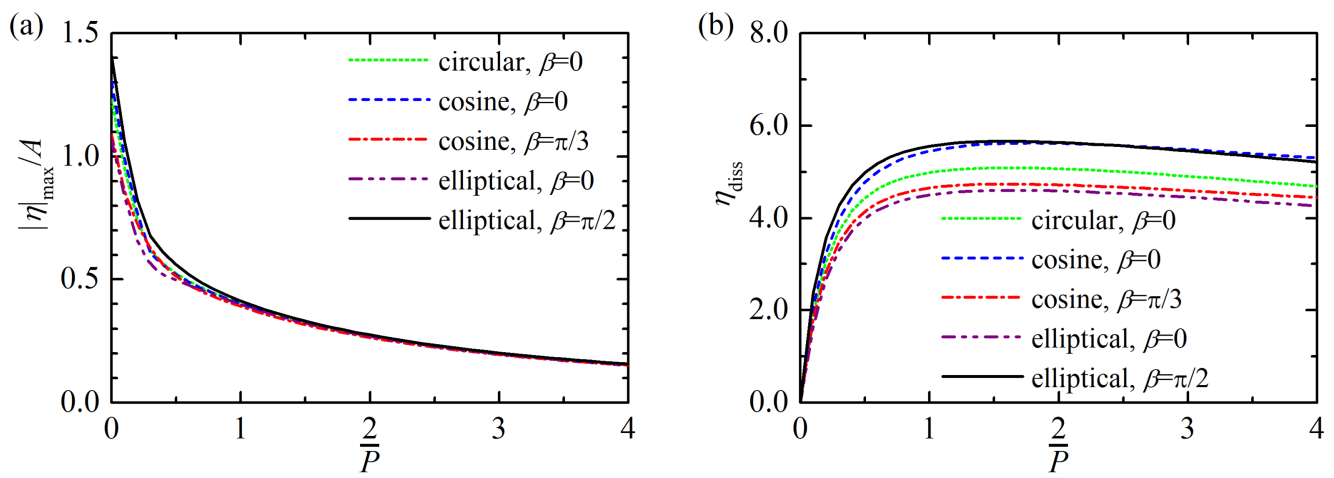

Figure 15: (a) Maximum deflection amplitude (i.e., $|\eta|_{\max } / A$ ) and (b) Power dissipation (i.e., $\left.\eta_{\text {diss }}\right)$ of the plates with a simply supported edge as functions of the porosity parameter, $\bar{P}$. $[k h=2.0, \bar{\chi}=\bar{\gamma}=0.01]$.

edge in the five cases in terms of deflection amplitude and the instantaneous deflection at $t=0$ for $\bar{P}=0$ (i.e., non-porous) and 1.0, respectively.

The deflection contour of the plate with a simply supported edge is different from that with a free edge. As shown in Figs. 16 and 17, the deflection vanishes at the edge of the plate for all the cases because of the displacement restriction. For a non-porous plate, i.e., $\bar{P}=0$, there are two separate internal regions of the plate where the response is stronger than the other regions (Fig. 16). The largest deflection occurs at the leeward internal region. When the plate is porous with $\bar{P}=1.0$ (Fig. 17), the two internal regions where the strong deflection occurred previously in a non-porous plate merge into one with its position moved waveward. This is reasonable because of the power dissipation induced by the porosity, which will reduce the response.

\subsubsection{Performance of the plate with a clamped edge}

In this subsection, the performance of the plate with a clamped edge is discussed. In this case, not only the plate displacement, but also the rotation at the edge is restrained. Figure 18 shows the variation of the maximum deflection amplitude and the wave power dissipation with the porosity parameter for different cases. 

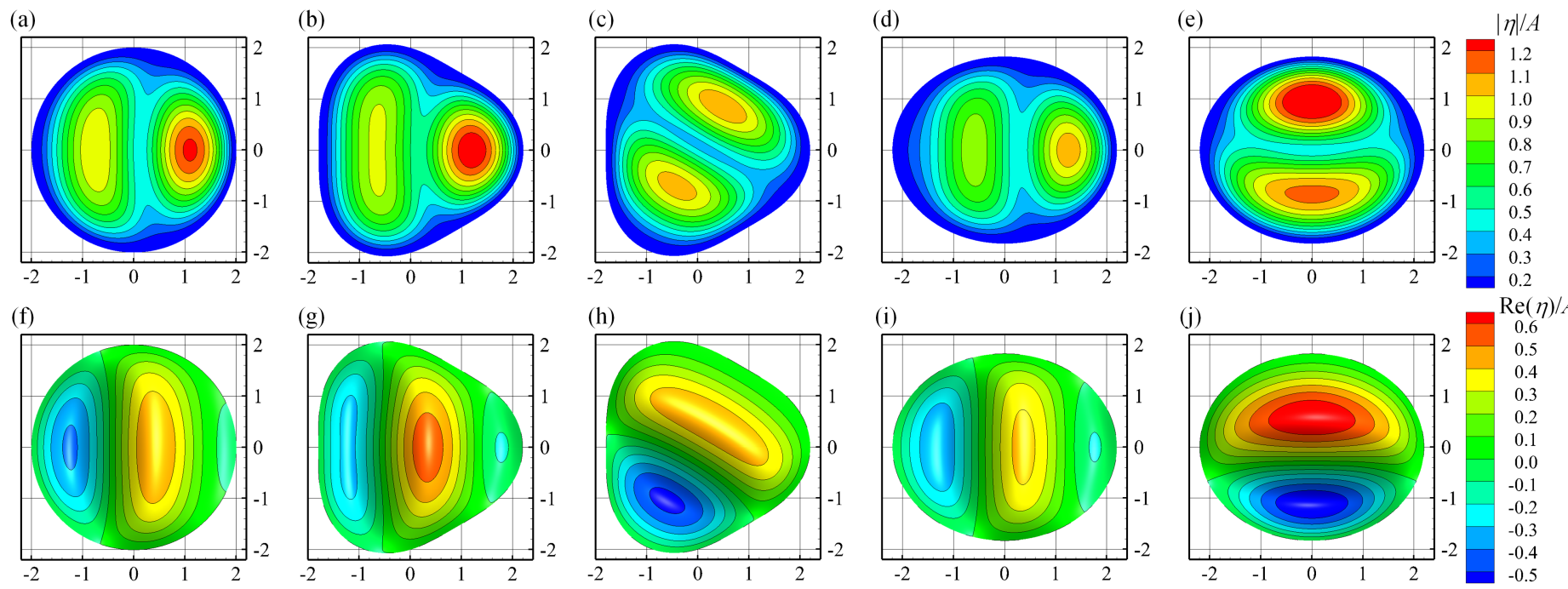

Figure 16: Deflection of the plate with a simply supported edge in different cases for $\bar{P}=0$, (a)-(e) deflection amplitude, $|\eta| / A$, and (f)-(j) instantaneous deflection at $t=0, \operatorname{Re}(\eta) / A$. $[k h=2.0, \bar{\chi}=\bar{\gamma}=0.01]$.

(a)

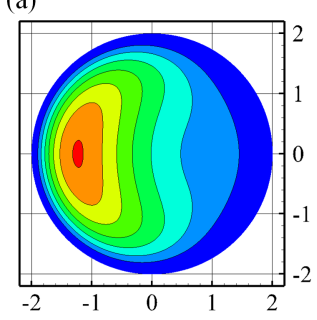

(f)

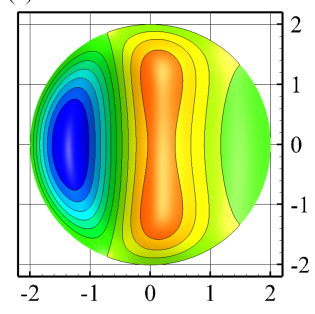

(b)

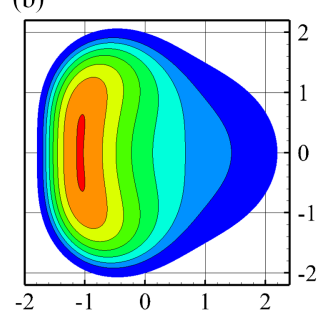

(g)

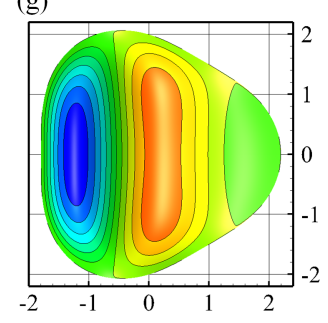

(c)

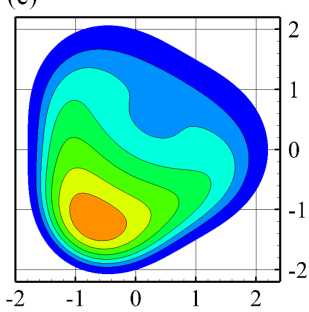

(h)

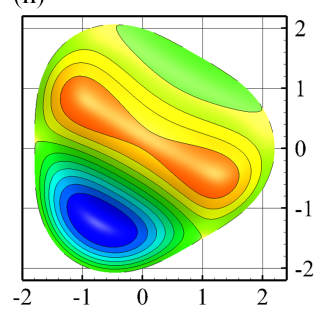

(d)

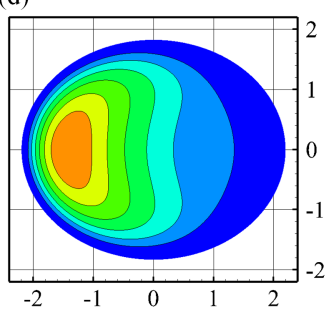

(i)

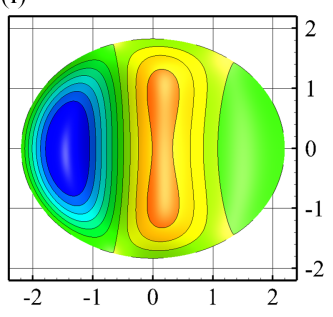

(e)

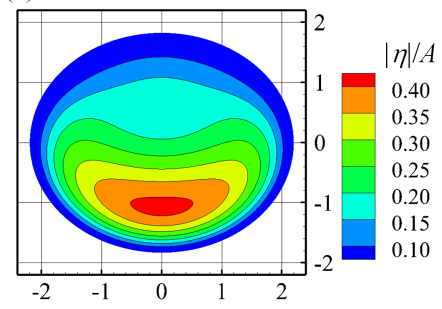

(j)

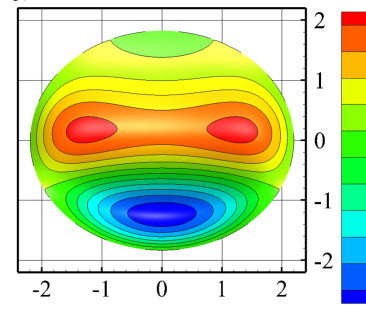

$\operatorname{Re}(\eta) / A$

0.20

0.15

0.05
0.00

-0.05
-0.10

$-0.10$

$-0.15$

-0.25
-0.30

Figure 17: Deflection of the plate with a simply supported edge in different cases for $\bar{P}=1.0$,

(a)-(e) deflection amplitude, $|\eta| / A$, and (f)-(j) instantaneous deflection at $t=0, \operatorname{Re}(\eta) / A$. $[k h=2.0, \bar{\chi}=\bar{\gamma}=0.01]$. 
The pattern of the variation of $|\eta|_{\max } / A$ for a clamped edge (Fig. 18a) with $\bar{P}$ is similar to that for a simply supported edge, and can be divided into two stages, i.e., stage one with $\bar{P}<0.5$, and stage two with $\bar{P}>0.5$. For the plate with a rather small value of $\bar{P}$, e.g., $\bar{P}<0.3$, the largest $|\eta|_{\max } / A$ for the plate with a clamped edge among the five cases examined occurs in case $2 \mathrm{a}$ (i.e., cosine shape with $\beta=0$ ), rather than in case $3 \mathrm{~b}$ (i.e., elliptical shape with $\beta=\pi / 2$ ) as investigated for both the free edge and simply supported edge conditions. The smallest $|\eta|_{\max } / A=0.10$ is obtained at $\bar{P}=4.0$.

As plotted in Fig. $18 \mathrm{~b}$, the peak of the $\eta_{\mathrm{diss}}-\bar{P}$ curve for the clamped edge occurs at around $\bar{P}=2.5$, which is larger than that for both the free edge $(\bar{P} \approx 1.0)$ and the simply supported cases $(\bar{P} \approx 1.6)$. Due to the more restrict constraints at the edge, the overall wave power dissipated by the plate with a clamped edge for $\bar{P}<3.0$ (Fig. $18 \mathrm{~b}$ ) is smaller than that dissipated by the plate with a simply supported edge (Fig. 15p). For the plate with a large value of porosity, e.g., $\bar{P}>3.5$, performance of the plate with a clamped edge in terms of power dissipation is almost the same as that with a simply supported edge. The plates in case $2 \mathrm{a}$ (i.e., cosine shape with $\beta=0$ ) and case $3 \mathrm{~b}$ (i.e., elliptical shape with $\beta=\pi / 2$ ) work similarly to one another and dissipate more wave energy than in the other cases for the whole computed range of $\bar{P}$.

The deflection contour of the plate with a clamped edge in the five cases in terms of deflection amplitude and the instantaneous deflection at $t=0$ for $\bar{P}=0$ (i.e., non-porous) and 1.0 can be found in Figs. 19 and 20 respectively, in which the pattern of the deflection distribution over the plate looks very similar to those of the plate with a simply supported edge (Figs. 16 and 17). Here the main differences between them are discussed. As expected, due to the displacement restriction, the deflection vanishes at the edge of the plate for all the cases, and the weak response region close to the edge is obviously broader than that for the plate with a simply supported edge because of the additional rotation restriction at the edge.

For a non-porous plate, i.e., $\bar{P}=0$ (Fig. 19 , although the edge of the plate is more strictly constrained, the deflection in certain regions of the plate can 

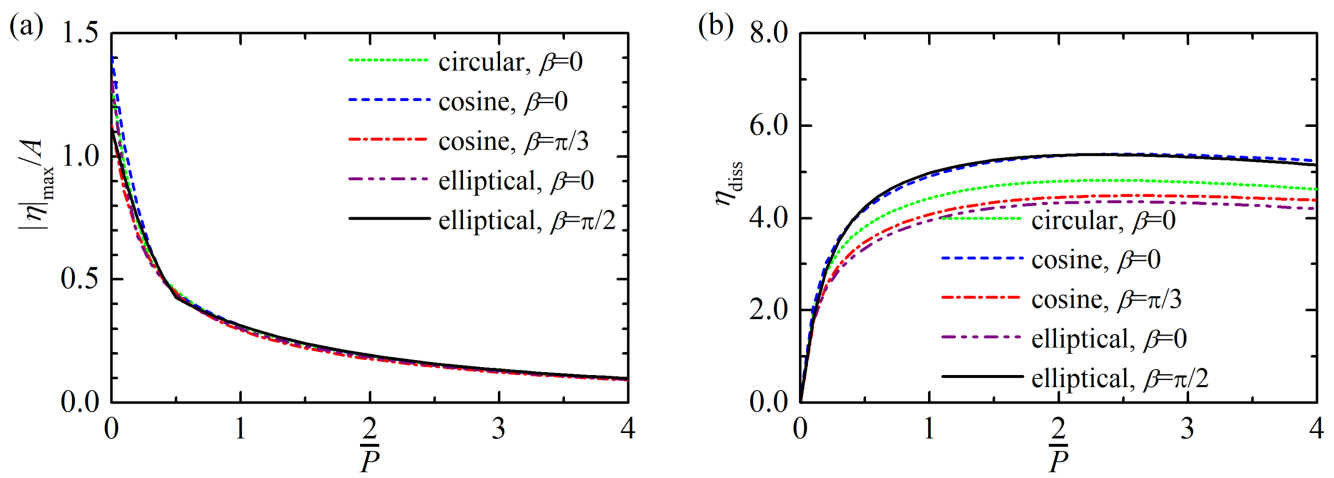

Figure 18: (a) Maximum deflection amplitude (i.e., $|\eta| \max / A$ ) and (b) Power dissipation (i.e., $\left.\eta_{\text {diss }}\right)$ of the plates with a clamped edge as functions of the porosity parameter, $\bar{P}$. $[k h=2.0$, $\bar{\chi}=\bar{\gamma}=0.01]$.

be larger in some cases than that for the simply supported edge condition (Fig. 16), e.g., case $3 a$ (elliptical shape with $\beta=0$ ). Unlike in the other cases, the

375 largest deflection for case $2 \mathrm{~b}$ (cosine shape with $\beta=\pi / 3$ ) occurs in the waveward internal region rather than the leeward one. For a porous plate with $\bar{P}=1.0$ (Fig. 20), the overall deflection is significantly reduced compared to $\bar{P}=0$ (Fig. 19), and is also smaller than the porous plate with a simply supported edge (Fig. 17). The peak and trough of the instantaneous deflection contour for $\bar{P}=0$ and 1.0 (Figs. 19 and 20 are more concentrated towards the plate centre in comparison with a simply supported edge (Figs. 16 and 17).

\section{Conclusions}

A semi-analytical model based on linear potential flow theory was developed to investigate water-wave interaction with a porous elastic plate with arbitrary shape floating on water of finite depth. The edge of the plate can be free, simply supported or clamped. In order to deal with the terms associated with the radius function and to solve the unknown coefficients of the spatial potentials, both the Fourier series expansion method and an eigenfunction-matching method were used. The energy dissipated by the plate due to the porosity is also calculated. 

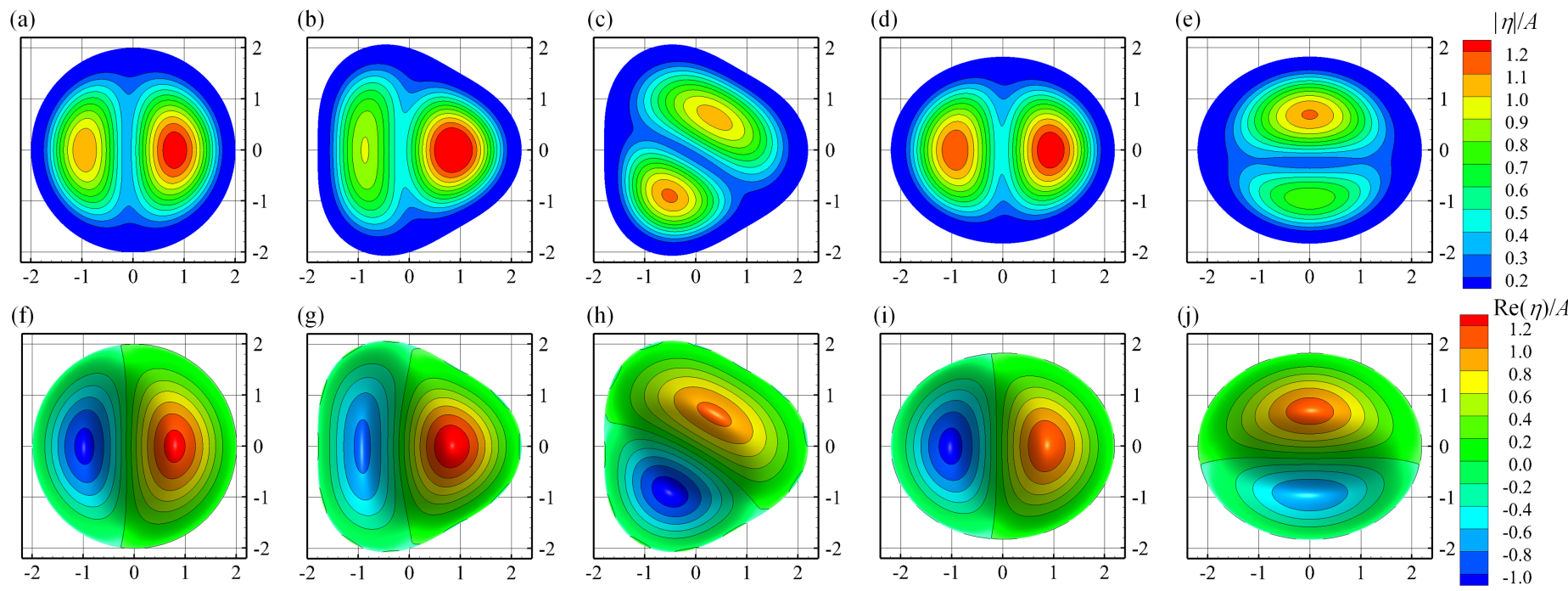

Figure 19: Deflection of the plate with a clamped edge in different cases for $\bar{P}=0$, (a)-(e) deflection amplitude, $|\eta| / A$, and (f)-(j) instantaneous deflection at $t=0, \operatorname{Re}(\eta) / A$. $[k h=2.0$, $\bar{\chi}=\bar{\gamma}=0.01]$.
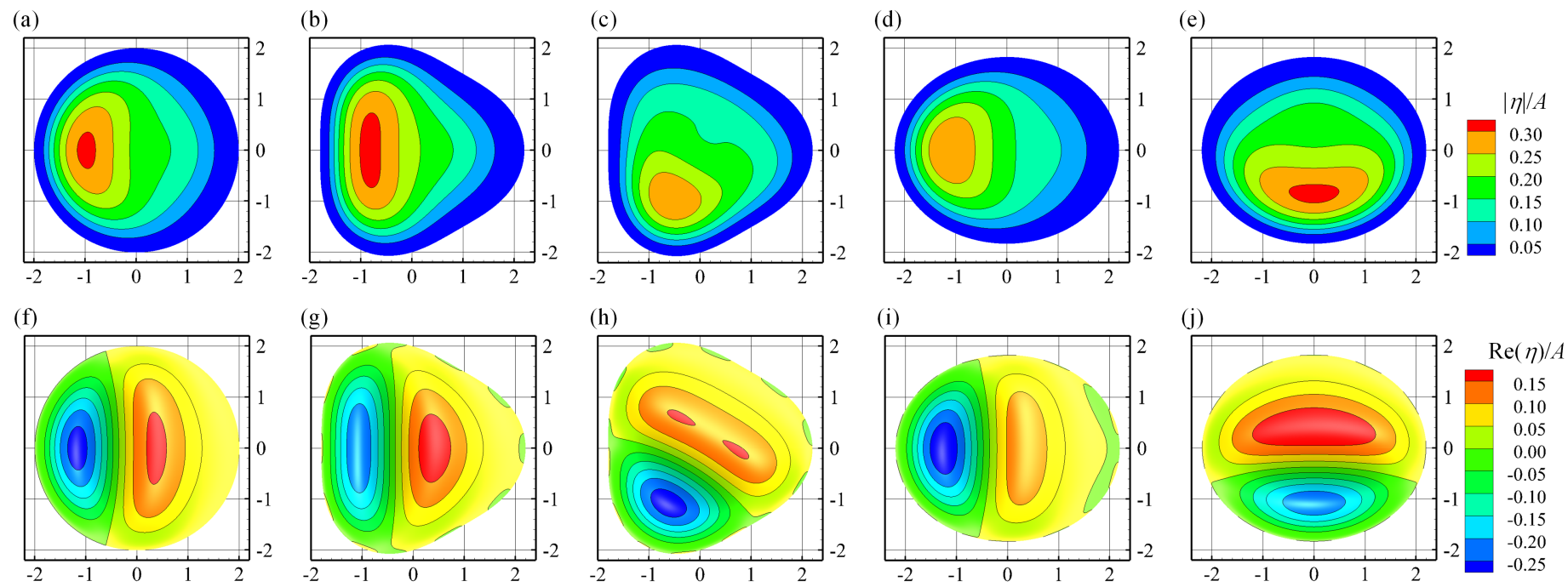

Figure 20: Deflection of the plate with a clamped edge in different cases for $\bar{P}=1.0$, (a)-(e) deflection amplitude, $|\eta| / A$, and (f)-(j) instantaneous deflection at $t=0, \operatorname{Re}(\eta) / A$. $[k h=2.0$, $\bar{\chi}=\bar{\gamma}=0.01]$. 

waveward side.

As $\bar{P}$ increases from 0 , the nondimensional energy dissipation, $\eta_{\text {diss }}$, first increases from 0 to a peak, and then decreases regardless of the shape of the plate. The $\bar{P}$ corresponding to the peak of $\eta_{\text {diss }}$ for a free edge and a clamped ${ }_{420}$ edge are the smallest and the largest, respectively, among the three different supported edge or a clamped edge, and a circular porous plate with a free edge, were carried out to validate the semi-analytical model. In both cases the results of the present model are in excellent agreement with the published data. The validated model is then applied to investigate performance of a plate with three different shapes, i.e., circular, cosine and elliptical with the same area, and with different kinds of edge conditions for $k h=2.0, \bar{\chi}=\bar{\gamma}=0.01$. The following conclusions may be drawn.

The maximum deflection amplitude of the plate shows a monotonic decrease as the porosity parameter becomes larger. For the plate with a free edge, the declining trend of $|\eta|_{\max } / A$ versus $\bar{P}$ is steady in the whole computed range of $\bar{P}$, whereas for the plate with a simply supported edge or a clamped edge, $|\eta|_{\max } / A$ for $\bar{P}<0.5$ is more sensitive to the change in $\bar{P}$ compared to the rest of the computed range of $\bar{P}$.

For the non-porous plate with a free edge, the deflection at both the waveward and leeward edges is larger than elsewhere. For $\bar{P}=1.0$, a strong response of the plate occurs at the waveward edge. The deflection vanishes at the edges of the plate with either a simply supported edge or a clamped edge in all cases, and the weak response region close to the edge for the clamped edge condition is broader than that for the simply supported edge.

For the non-porous plate with either a simply supported edge or a clamped edge, there are two separate internal regions of the plate where the response is stronger than in the other regions, and the largest deflection occurs at the leeward internal region for all the cases, except case $2 \mathrm{~b}$ (cosine shape, $\beta=\pi / 3$ ) with a clamped edge. For $\bar{P}=1.0$, the two internal regions where the strong deflection occurred previously in a non-porous plate merge into one on the

Two case studies, i.e., an elliptical non-porous plate with either a simply 
kinds of edge conditions. The power dissipated by an elliptical plate with its major axis perpendicular to the incident wave direction is the largest among the case studies for the majority of the porosity values tested. The overall wave power dissipated by the plate with either a simply supported or clamped edge is smaller than that dissipated by the plate with a free edge.

The semi-analytical model may not be suitable for extreme wave-structure interactions since viscous effects are not captured in the framework of linear potential flow theory. This paper deals with an isolated floating porous elastic plate. For an array of these plates with small gaps between them, the hydrodynamic interactions between the plates may play a significant role in their overall response and wave energy dissipation. The present semi-analytical model can be extended to study the water-wave scattering by an array of floating porous elastic plates, which will be reported elsewhere.

\section{Acknowledgements}

The research was supported by Intelligent Community Energy (ICE), INTERREG V FCE, European Commission (Contract No. 5025). The corresponding author wishes to thank Dr Feng Xu from the University of Birmingham for valuable discussions.

\section{Appendix A. The full expressions of $M$ and $V$ at the edge of the

$$
\begin{aligned}
\left.M\right|_{r=R}= & {\left.\left[\Delta \eta-(1-v)\left(\partial_{s}^{2} \eta+\frac{\mathrm{d} \alpha}{\mathrm{d} s} \partial_{n} \eta\right)\right]\right|_{r=R} } \\
= & \frac{R^{2}+v R^{\prime 2}}{R^{2}+R^{\prime 2}} \frac{\partial^{2} \eta}{\partial r^{2}}+\frac{2(v-1) R^{\prime}}{R^{2}+R^{\prime 2}} \frac{\partial^{2} \eta}{\partial r \partial \theta}+\frac{v R^{2}+R^{\prime 2}}{R^{2}\left(R^{2}+R^{\prime 2}\right)} \frac{\partial^{2} \eta}{\partial \theta^{2}} \\
& +\frac{v R^{2}+R^{\prime 2}}{R\left(R^{2}+R^{\prime 2}\right)} \frac{\partial \eta}{\partial r}+\frac{2(1-v) R^{\prime}}{R\left(R^{2}+R^{\prime 2}\right)} \frac{\partial \eta}{\partial \theta},
\end{aligned}
$$


in which $v$ is Poisson ratio. $v=0.3$ is employed in this paper.

$$
\begin{aligned}
& \left.V\right|_{r=R}=\left.\left(\partial_{n} \Delta \eta+(1-v) \partial_{s} \partial_{n} \partial_{s} \eta\right)\right|_{r=R} \\
& =\frac{R\left[R^{2}+(2-v) R^{\prime 2}\right]}{\left(R^{2}+R^{\prime 2}\right)^{1.5}} \frac{\partial^{3} \eta}{\partial r^{3}}+\frac{R^{\prime}\left[(1-2 v) R^{2}+(v-2) R^{\prime 2}\right]}{R\left(R^{2}+R^{\prime 2}\right)^{1.5}} \frac{\partial^{3} \eta}{\partial r^{2} \partial \theta} \\
& +\frac{(2-v) R^{2}+(2 v-1) R^{\prime 2}}{R\left(R^{2}+R^{\prime 2}\right)^{1.5}} \frac{\partial^{3} \eta}{\partial r \partial \theta^{2}}+\frac{R^{\prime}\left[(v-2) R^{2}-R^{\prime 2}\right]}{R^{3}\left(R^{2}+R^{\prime 2}\right)^{1.5}} \frac{\partial^{3} \eta}{\partial \theta^{3}} \\
& +\frac{R^{4}+2 v R^{2} R^{\prime 2}+R^{\prime 4}+(1-v) R\left(R^{2}-R^{\prime 2}\right) R^{\prime \prime}}{\left(^{2}+R^{\prime 2}\right)^{2.5}} \frac{\partial^{2} \eta}{\partial r^{2}} \\
& +\frac{R^{\prime}\left[(3 v-4) R^{4}+4(v-1) R^{3} R^{\prime \prime}+(1-3 v) R^{2} R^{\prime 2}+(1-2 v) R^{\prime 4}\right]}{R^{2}\left(R^{2}+R^{\prime 2}\right)^{2.5}} \frac{\partial^{2} \eta}{\partial r \partial \theta} \\
& +\frac{\left[(v-3) R^{4}+(v-1) R^{3} R^{\prime \prime}-(3 v+1) R^{2} R^{\prime 2}+(1-v) R R^{\prime 2} R^{\prime \prime}-2 v R^{\prime 4}\right]}{R^{2}\left(R^{2}+R^{\prime 2}\right)^{2.5}} \frac{\partial^{2} \eta}{\partial \theta^{2}} \\
& -\frac{R^{4}+2 v R^{2} R^{\prime 2}+R^{\prime 4}+(1-v) R R^{\prime \prime}\left(R^{2}-R^{\prime 2}\right)}{R\left(R^{2}+R^{\prime 2}\right)^{2.5}} \frac{\partial \eta}{\partial r} \\
& +\frac{2(1-v) R^{\prime}\left[R^{4}-2 R^{2} R^{\prime 2}-R^{\prime 4}+2 R^{3} R^{\prime \prime}\right]}{R^{3}\left(R^{2}+R^{\prime 2}\right)^{2.5}} \frac{\partial \eta}{\partial \theta}
\end{aligned}
$$

where a typo of the $\partial^{3} \eta / \partial \theta^{3}$ item as derived by $\mathrm{Xu}$ and $\mathrm{Lu}(2011)$ has been corrected.

\section{Appendix B. Derivation process of the formulas and calculation for} the unknown coefficients $A_{m, l}$ and $B_{m, l}$

Inserting the expression of the spatial potentials, as given in Eqs. 10 and (12), into the continuity boundary conditions, i.e., Eqs. (16) and (17), gives:

$$
\begin{aligned}
-\frac{\mathrm{i} g A}{\omega} Z_{0}(z) & \sum_{m=-\infty}^{\infty} \mathrm{i}^{m} \mathrm{e}^{-\mathrm{i} m \beta} J_{m}\left(k_{0} R\right) \mathrm{e}^{\mathrm{i} m \theta}+\sum_{m=-\infty}^{\infty} \sum_{l=0}^{\infty} A_{m, l} H_{m}\left(k_{l} R\right) Z_{l}(z) \mathrm{e}^{\mathrm{i} m \theta} \\
= & \sum_{m=-\infty}^{\infty} \sum_{l=-2}^{\infty} B_{m, l} J_{m}\left(\kappa_{l} R\right) Y_{l}(z) \mathrm{e}^{\mathrm{i} m \theta}, \quad-h<z<0,
\end{aligned}
$$




$$
\begin{aligned}
& -\frac{\mathrm{i} g A}{\omega} Z_{0}(z) \sum_{m=-\infty}^{\infty} \mathrm{i}^{m} \mathrm{e}^{-\mathrm{i} m \beta}\left(R^{2} k_{0} J_{m}^{\prime}\left(k_{0} R\right)-\mathrm{i} m R^{\prime} J_{m}\left(k_{0} R\right)\right) \mathrm{e}^{\mathrm{i} m \theta} \\
& +\sum_{m=-\infty}^{\infty} \sum_{l=0}^{\infty} A_{m, l}\left(R^{2} k_{l} H_{m}^{\prime}\left(k_{l} R\right)-\mathrm{i} m R^{\prime} H_{m}\left(k_{l} R\right)\right) Z_{l}(z) \mathrm{e}^{\mathrm{i} m \theta} \\
& =\sum_{m=-\infty}^{\infty} \sum_{l=-2}^{\infty} B_{m, l}\left(R^{2} \kappa_{l} J_{m}^{\prime}\left(\kappa_{l} R\right)-\mathrm{i} m R^{\prime} J_{m}\left(\kappa_{l} R\right)\right) Y_{l}(z) \mathrm{e}^{\mathrm{i} m \theta}, \quad-h<z<0 .
\end{aligned}
$$

To solve the wave scattering problem with an eigenfunction-matching method, the functions relating to the radius function of the plate edge, i.e., $r=R$, are expanded into a Fourier series as follows (Yu et al. 2019; Zheng et al., 2019b):

$$
J_{m}\left(k_{0} R\right)=\sum_{q=-\infty}^{\infty} f_{m, q}^{J} \mathrm{e}^{\mathrm{i} q \theta} ; \quad R^{2} k_{0} J_{m}^{\prime}\left(k_{0} R\right)-\mathrm{i} m R^{\prime} J_{m}\left(k_{0} R\right)=\sum_{q=-\infty}^{\infty} f_{m, q}^{J^{\prime}} \mathrm{e}^{\mathrm{i} q \theta},
$$

$H_{m}\left(k_{l} R\right)=\sum_{q=-\infty}^{\infty} f_{m, l, q}^{H} \mathrm{e}^{\mathrm{i} q \theta} ; \quad R^{2} k_{l} H_{m}^{\prime}\left(k_{l} R\right)-\mathrm{i} m R^{\prime} H_{m}\left(k_{l} R\right)=\sum_{q=-\infty}^{\infty} f_{m, l, q}^{H^{\prime}} \mathrm{e}^{\mathrm{i} q \theta}$,

$J_{m}\left(\kappa_{l} R\right)=\sum_{q=-\infty}^{\infty} f_{m, l, q}^{J} \mathrm{e}^{\mathrm{i} q \theta} ; \quad R^{2} \kappa_{l} J_{m}^{\prime}\left(\kappa_{l} R\right)-\mathrm{i} m R^{\prime} J_{m}\left(\kappa_{l} R\right)=\sum_{q=-\infty}^{\infty} f_{m, l, q}^{J^{\prime}} \mathrm{e}^{\mathrm{i} q \theta}$,

in which the Fourier coefficients, i.e., $f_{m, q}^{J}, f_{m, q}^{J^{\prime}}, f_{m, l, q}^{H}, f_{m, l, q}^{H^{\prime}}, f_{m, l, q}^{J}$, and $f_{m, l, q}^{J^{\prime}}$, can be obtained using:

$$
\chi_{q}=\frac{1}{2 \pi} \int_{0}^{2 \pi} \psi(\theta) \mathrm{e}^{-\mathrm{i} q \theta} \mathrm{d} \theta
$$

where $\chi_{q}$ and $\psi(\theta)$ represent the Fourier coefficients and functions to be expanded, respectively. 
By means of these Fourier coefficients, Eqs. B.1)-(B.2) can be rewritten as

$$
\begin{gathered}
-\frac{\mathrm{i} g A}{\omega} \sum_{m=-\infty}^{\infty} \mathrm{i}^{m} \mathrm{e}^{-\mathrm{i} m \beta} \sum_{q=-\infty}^{\infty} f_{m, q}^{J} \mathrm{e}^{\mathrm{i} q \theta}+\sum_{m=-\infty}^{\infty} \sum_{l=0}^{\infty} A_{m, l} \sum_{q=-\infty}^{\infty} f_{m, l, q}^{H} \mathrm{e}^{\mathrm{i} q \theta} Z_{l}(z) \mathrm{e}^{\mathrm{i} m \theta} \\
=\sum_{m=-\infty}^{\infty} \sum_{l=-2}^{\infty} B_{m, l} \sum_{q=-\infty}^{\infty} f_{m, l, q}^{J} \mathrm{e}^{\mathrm{i} \varphi \theta} Y_{l}(z) \mathrm{e}^{\mathrm{i} m \theta}, \quad-h<z<0,
\end{gathered}
$$

$$
\begin{gathered}
-\frac{\mathrm{i} g A}{\omega} \sum_{m=-\infty}^{\infty} \mathrm{i}^{m} \mathrm{e}^{-\mathrm{i} m \beta} \sum_{q=-\infty}^{\infty} f_{m, q}^{J^{\prime}} \mathrm{e}^{\mathrm{i} q \theta}+\sum_{m=-\infty}^{\infty} \sum_{l=0}^{\infty} A_{m, l} \sum_{q=-\infty}^{\infty} f_{m, l, q}^{H^{\prime}} \mathrm{e}^{\mathrm{i} q \theta} Z_{l}(z) \mathrm{e}^{\mathrm{i} m \theta} \\
=\sum_{m=-\infty}^{\infty} \sum_{l=-2}^{\infty} B_{m, l} \sum_{q=-\infty}^{\infty} f_{m, l, q}^{J^{\prime}} \mathrm{e}^{\mathrm{i} q \theta} Y_{l}(z) \mathrm{e}^{\mathrm{i} m \theta}, \quad-h<z<0 .
\end{gathered}
$$

Multiplying both sides of Eqs. B.5- B.6 with $Z_{\zeta}(z) \mathrm{e}^{-\mathrm{i} \tau \theta}$ and integrating in $z \in[-h, 0]$ and $\theta \in[0,2 \pi]$, using their orthogonality characteristics, we get, for any pair of integers $(\tau, \zeta)$ :

$$
\begin{array}{r}
\sum_{m=-\infty}^{\infty} A_{m, \zeta} f_{m, \zeta,(\tau-m)}^{H} A_{\zeta}-\sum_{m=-\infty}^{\infty} \sum_{l=-2}^{\infty} B_{m, l} f_{m, l,(\tau-m)}^{J} Y_{l, \zeta} \\
=\frac{\mathrm{i} g A}{\omega} A_{0} \delta_{0, \zeta} \sum_{m=-\infty}^{\infty} \mathrm{i}^{m} \mathrm{e}^{-\mathrm{i} m \beta} f_{m,(\tau-m)}^{J} \\
\sum_{m=-\infty}^{\infty} A_{m, \zeta} f_{m, \zeta,(\tau-m)}^{H^{\prime}} A_{\zeta}-\sum_{m=-\infty}^{\infty} \sum_{l=-2}^{\infty} B_{m, l} f_{m, l,(\tau-m)}^{J^{\prime}} Y_{l, \zeta} \\
=\frac{\mathrm{i} g A}{\omega} A_{0} \delta_{0, \zeta} \sum_{m=-\infty}^{\infty} \mathrm{i}^{m} \mathrm{e}^{-\mathrm{i} m \beta} f_{m,(\tau-m)}^{J^{\prime}}
\end{array}
$$

where

$$
\begin{gathered}
A_{l}=\int_{-h}^{0} Z_{l}^{2}(z) \mathrm{d} z=\frac{1}{2}\left(\frac{\sinh \left(k_{l} h\right) \cosh \left(k_{l} h\right)+k_{l} h}{k_{l} \cosh ^{2}\left(k_{l} h\right)}\right) \\
Y_{l, \zeta}=\int_{-h}^{0} Y_{l}(z) Z_{\zeta}(z) \mathrm{d} z=\frac{\kappa_{l} \sinh \left(\kappa_{l} h\right) \cosh \left(k_{\zeta} h\right)-k_{\zeta} \cosh \left(\kappa_{l} h\right) \sinh \left(k_{\zeta} h\right)}{\left(\kappa_{l}^{2}-k_{\zeta}^{2}\right) \cosh \left(\kappa_{l} h\right) \cosh \left(k_{\zeta} h\right)} .
\end{gathered}
$$


Similarly, the edge boundary conditions, Eq. (9), can be appended to the system to close it. Here we take a clamped edge as an example: after inserting Eq. (15) into Eq. (9a) and making some rearrangements, we have

$$
\sum_{m=-\infty}^{\infty} \sum_{l=-2}^{\infty} \frac{B_{m, l} f_{m, l,(\tau-m)}^{J}}{\chi \kappa_{l}^{4}+1-\left(\omega^{2} / g\right) \gamma}=0, \quad \sum_{m=-\infty}^{\infty} \sum_{l=-2}^{\infty} \frac{B_{m, l} f_{m, l,(\tau-m)}^{J^{\prime}}}{\chi \kappa_{l}^{4}+1-\left(\omega^{2} / g\right) \gamma}=0
$$

In order to evaluate the unknown coefficients $A_{m, l}$ and $B_{m, l}$, we take $(2 M+1)$ terms $(m=-M, \ldots, 0, \ldots, M),(L+1)$ terms $(l=0,1, \ldots, L)$ for $A_{m, l}$, and $(L+3)$ terms $(l=-2,-1,0,1, \ldots, L)$ for $B_{m, l}$, respectively, and take $(\tau=$ $-M, \ldots, 0, \ldots, M)$ and $(\zeta=0,1, \ldots, L)$ in Eqs. (B.7), (B.8) and (B.11), thus a $2(2 M+1)(L+2)$ order complex linear equation matrix is obtained, with the same number of unknown coefficients. Convergence analysis of the semianalytical model in terms of the angular and vertical cutoffs, i.e., $M$ and $L$, is given in Section 5.1

\section{References}

Chen, X., Chen, Q., Zhan, J., Liu, D., 2016. Numerical simulations of wave propagation over a vegetated platform. Coastal Engineering 110, $64-75$.

Cheng, L.H., Fen, C.Y., Li, Y.H., Jiang, W.Y., 2013. Experimental study on a new type floating breakwater, in: Proceedings of the 7th International Conference on Asian and Pacific Coasts, pp. 24-26.

Cho, I.H., Kim, M.H., 1998. Interactions of a horizontal flexible membrane with oblique waves. Journal of Fluid Mechanics 367, 139-161.

Cho, I.H., Kim, M.H., 2000. Interactions of horizontal porous flexible membrane with waves. Journal of Waterway, Port, Coastal, and Ocean Engineering 126, $245-253$.

Chwang, A.T., Chan, A.T., 1998. Interaction between porous media and wave motion. Annual Review of Fluid Mechanics 30, 53-84. 
Chwang, A.T., Wu, J., 1994. Wave scattering by submerged porous disk. Journal of Engineering Mechanics 120, 2575-2587.

Duncan, T., 2014. Aqua biofilter ${ }^{\mathrm{TM}}$ floating wetlands \& floating islands. URL: http://www . aquabiofilter.com/

Evans, D.V., Peter, M.A., 2011. Asymptotic reflection of linear water waves by submerged horizontal porous plates. Journal of Engineering Mathematics 69, $135-154$.

Farina, L., Martin, P., 1998. Scattering of water waves by a submerged disc using a hypersingular integral equation. Applied Ocean Research 20, 121 134 .

Gayen, R., Mondal, A., 2014. A hypersingular integral equation approach to the porous plate problem. Applied Ocean Research 46, 70 - 78 .

Kagemoto, H., Yue, D., 1986. Interactions among multiple three-dimensional bodies in water waves: an exact algebraic method. Journal of Fluid Mechanics 166, 189-209.

Kamble, R., Patil, D., 2012. Artificial floating island: solution to river water pollution in india. case study: rivers in pune city, in: Proceedings of the International Conference on Environmental, Biomedical and Biotechnology, Dubai, UAE, pp. 136-140.

Kohout, A.L., Meylan, M.H., Sakai, S., Hanai, K., Leman, P., Brossard, D., 2007. Linear water wave propagation through multiple floating elastic plates of variable properties. Journal of Fluids and Structures 23, 649-663.

Koley, S., Mondal, R., Sahoo, T., 2018. Fredholm integral equation technique for hydroelastic analysis of a floating flexible porous plate. European Journal of Mechanics - B/Fluids 67, $291-305$.

Kundu, S., Datta, R., Gayen, R., Islam, N., 2019. The interaction of flexural495 gravity waves with a submerged rigid disc. Applied Ocean Research 92, 101912. 
Liu, Y., Li, Y., 2011. An alternative analytical solution for water-wave motion over a submerged horizontal porous plate. Journal of Engineering Mathematics $69,385-400$.

Martin, P.A., Farina, L., 1997. Radiation of water waves by a heaving submerged horizontal disc. Journal of Fluid Mechanics 337, 365-379.

Meylan, M.H., 2002. Wave response of an ice floe of arbitrary geometry. Journal of Geophysical Research 107, 3005.

Meylan, M.H., Bennetts, L.G., Peter, M.A., 2017. Water-wave scattering and energy dissipation by a floating porous elastic plate in three dimensions. Wave Motion 70, 240-250.

Meylan, M.H., Squire, V.A., 1996. Response of a circular ice floe to ocean waves. Journal of Geophysical Research 101, 8869-8884.

Mohapatra, S.C., Sahoo, T., Guedes Soares, C., 2018. Surface gravity wave interaction with a submerged horizontal flexible porous plate. Applied Ocean Research 78, 61-74.

Montiel, F., Bennetts, L.G., Squire, V.A., Bonnefoy, F., Ferrant, P., 2013a. Hydroelastic response of floating elastic discs to regular waves. part 2. modal analysis. Journal of Fluid Mechanics 723, 629-652.

Montiel, F., Bonnefoy, F., Ferrant, P., Bennetts, L.G., Squire, V.A., Marsault, P., 2013b. Hydroelastic response of floating elastic discs to regular waves. part 1. wave basin experiments. Journal of Fluid Mechanics 723, 604-628.

Peter, M.A., Meylan, M.H., Chung, H., 2004. Wave scattering by a circular elastic plate in water of finite depth: a closed form solution. International Journal of Offshore and Polar Engineering 14, 81-85.

Praveen, K.M., Karmaker, D., Guedes Soares, C., 2018. Hydroelastic analysis of articulated floating elastic plate based on timoshenko-mindlin plate theory. Ships and Offshore Structures 13, 287-301. 
Press, W.H., Teukolsky, S.A., Vetterling, W.T., Flannery, B.P., 2007. Numerical recipes: The art of scientific computing. Cambridge University Press.

Reddy, J., 2007. Theory and analysis of elastic plates and shells. CRC Press.

Renzi, E., 2016. Hydroelectromechanical modelling of a piezoelectric wave energy converter. Proceedings of the Royal Society A-Mathematical Physical and Engineering Sciences 472, 20160715.

Sahoo, T., Yip, T.L., Chwang, A.T., 2001. Scattering of surface waves by a semi-infinite floating elastic plate. Physics of Fluids 13, 3215.

Squire, V.A., 2008. Synergies between vlfs hydroelasticity and sea-ice research, in: The Eighteenth International Offshore and Polar Engineering Conference, International Society of Offshore and Polar Engineers. pp. 1-13.

${ }_{535}$ Timoshenko, S., Woinowsky-Krieger, S., 1959. Theory of plates and shells. McGraw-Hill Book Company.

Wang, C.M., Tay, Z.Y., Takagi, K., Utsunomiya, T., 2010. Literature review of methods for mitigating hydroelastic response of vlfs under wave action. Applied Mechanics Reviews 63, 030802.

${ }_{540} \mathrm{Xu}, \mathrm{F} ., \mathrm{Lu}, \mathrm{D} ., 2011$. Hydroelastic interaction between water waves and a thin elastic plate of arbitrary geometry. Science China Physics, Mechanics \& Astronomy 54, 59-66.

Yip, T.L., Chwang, A.T., 1998. Water wave control by submerged pitching porous plate. Journal of Engineering Mechanics 124, 428-434.

${ }_{545}$ Yu, H., Zheng, S., Zhang, Y., Iglesias, G., 2019. Wave radiation from a truncated cylinder of arbitrary cross section. Ocean Engineering 173, 519 - 530.

Yu, X., Chwang, A.T., 1994. Water waves above submerged porous plate. Journal of Engineering Mechanics 120, 1270-1280. 
Zheng, S., Zhang, Y., 2018. Theoretical modelling of a new hybrid wave energy converter in regular waves. Renewable Energy 128, 125 - 141.

Zheng, S., Zhang, Y., Iglesias, G., 2018. Wave--structure interaction in hybrid wave farms. Journal of Fluids and Structures 83, $386-412$.

Zheng, S., Zhang, Y., Iglesias, G., 2019a. Coast/breakwater-integrated OWC: A theoretical model. Marine Structures 66, 121 - 135.

${ }_{555}$ Zheng, S., Zhang, Y., Liu, Y., Iglesias, G., 2019b. Wave radiation from multiple cylinders of arbitrary cross sections. Ocean Engineering 184, 11-22.

Zilman, G., Miloh, T., 2000. Hydroelastic buoyant circular plate in shallow water: a closed form solution. Applied Ocean Research 22, 191-198. 